\title{
On the Best Possible Competitive Ratio for the Multislope Ski-Rental Problem
}

\author{
Hiroshi Fujiwara
}

\author{
Takuma Kitano
}

\author{
Toshihiro Fujito
}

\begin{abstract}
The multislope ski-rental problem is an extension of the classical ski-rental problem, where the player has several lease options besides the pure rent and buy options. In this problem the hardness of an instance, which is the setting of options, significantly affects the player's performance. There is an algorithm that for a given instance, computes the best possible strategy. However, the output is given as numerical values and therefore the relational nature between an instance and the best possible performance for it has not been known. In this paper we prove that even for the easiest instance, a competitive ratio smaller than $e /(e-1) \approx 1.58$ cannot be achieved. More precisely, a tight lower bound on the best possible performance is obtained in a closed form parametrized by the number of options. Furthermore, we establish a matching upper and lower bound on the competitive ratio each for the 3-option and 4-option problems.
\end{abstract}

\section{Introduction}

In the classical ski-rental problem [KMRS88], the player decides either to rent a ski set or buy one, each time he/she goes skiing. For example, a store may offer the following options for ski sets: Rent for $\$ 50$ per day or buy for $\$ 500$. Each of the player's decisions has to be made in an online manner where he/she has no information on how many times he/she is going skiing from now and on. The objective is to minimize the total cost.

The multislope ski-rental problem [AIS08, LPSR08] is an extension of the classical ski-rental problem, where the player has several lease options of paying both of a per-time fee and an initial fee, in addition to the pure rent and buy options. The store may offer, other than the rest and buy options above, the following two lease options: Lease for $\$ 30$ per day with an initial fee of $\$ 100$, or lease for $\$ 15$ per day with an initial fee of $\$ 250$.

We refer to a set of such options as an instance, denoted by a pair of two vectors $(\boldsymbol{r}, \boldsymbol{b})$. The vector $\boldsymbol{r}$ indicates the per-time fee of each option and the vector $\boldsymbol{b}$ tells the fee of switching options. (The detailed definition shall appear in Section 2.) A strategy of the player specifies when and to which option to switch. According to the standard definition, we say that a strategy has a competitive ratio of $c$ if the player along the strategy is charged at most $c$ times the optimal offline cost, i.e., one with the number of times of skiing known in advance. For a given instance $(\boldsymbol{r}, \boldsymbol{b})$, we define the best possible competitive ratio $\tilde{c}(\boldsymbol{r}, \boldsymbol{b})$ as the minimum value of the competitive ratios for all possible strategies for $(\boldsymbol{r}, \boldsymbol{b})$. In short, $\tilde{c}(\boldsymbol{r}, \boldsymbol{b})$ is the competitive ratio of the best possible strategy for $(\boldsymbol{r}, \boldsymbol{b})$.

The numerical value of $\tilde{c}(\boldsymbol{r}, \boldsymbol{b})$ can be calculated by an algorithm of Augustine et al. [AIS08]. However, almost nothing has been known of dependencies between $(\boldsymbol{r}, \boldsymbol{b})$ and $\tilde{c}(\boldsymbol{r}, \boldsymbol{b})$. In this

\footnotetext{
${ }^{0} \mathrm{~A}$ preliminary version appeared in the 22nd Annual International Symposium on Algorithms and Computation (ISAAC), 2011. This work was supported by KAKENHI (23700014 and 23500014). The final publication is available at www.springerlink.com: http://dx.doi.org/10.1007/s10878-014-9762-9.
} 
paper we analyze $\inf \tilde{c}(\boldsymbol{r}, \boldsymbol{b})$ and $\sup \tilde{c}(\boldsymbol{r}, \boldsymbol{b})$ over reasonable instances, revealing the easiest and the hardest instances. Notice here that the supremum coincides with the matching upper and lower bound on the competitive ratio in the standard sense.

The analysis of the infimum is motivated by a realistic application: This problem can be regarded as Dynamic Power Management [ISG03] on a system that has multiple energy-saving states, for example, a Windows computer with Stand By state, Hibernate state, and so on. The objective is to minimize the energy consumption during an idle period when there is no user response. A pair of a strategy and an instance that achieve the infimum present the best specification of energy-saving states and the best state-transition schedule.

\subsection{Our Contribution}

Our results are summarized as follows:

(I) For integer $k \geq 2$, let $I(k)$ denote the entire set of instances, each consisting of exactly $k+1$ options. We here mean by "exactly" that there is no redundant option in each instance. (See Section 2 for the detailed settings.) For fixed $k$, we prove that $\inf \{\tilde{c}(\boldsymbol{r}, \boldsymbol{b}) \mid(\boldsymbol{r}, \boldsymbol{b}) \in I(k)\}=$ $\frac{(k+1)^{k}}{(k+1)^{k}-k^{k}}$. The infimum value monotonically decreases as $k$ grows. For example, 1.80 for $k=2$, 1.73 for $k=3$, and 1.70 for $k=4$. We have as corollary that $\inf \{\tilde{c}(\boldsymbol{r}, \boldsymbol{b}) \mid(\boldsymbol{r}, \boldsymbol{b}) \in I(k), k \geq$ $2\}=\frac{e}{e-1} \approx 1.58$. Our results are interpreted into the context of Dynamic Power Management as follows: The more energy-saving states are available, the better energy saving performance can be achieved. Nevertheless, there is a limit of improvement.

(II) We $\operatorname{show} \sup \{\tilde{c}(\boldsymbol{r}, \boldsymbol{b}) \mid(\boldsymbol{r}, \boldsymbol{b}) \in I(2)\} \approx 2.47$ and $\sup \{\tilde{c}(\boldsymbol{r}, \boldsymbol{b}) \mid(\boldsymbol{r}, \boldsymbol{b}) \in I(3)\} \approx 2.75$. As we have already mentioned, the supremum is the matching upper and lower bound on the competitive ratio in the ordinary sense. Our results are the first to establish a matching bound for the competitive ratio of the problem; it has only been known that an upper bound of 4 and a lower bound of 3.62 hold when arbitrary number of options are available [BCN00, Dam03]. In most studies, an upper bound and a lower bound have been separately analyzed. In contrast, our analysis is based on a quite different technique in the sense that we seek both bounds in the same time. The results in (I) and (II) are illustrated in Figure 1.

(III) We consider two subclasses of instances $I_{A}(k)$ and $I_{I}(k)$ which consist of additive instances and investment instances, respectively. For an additive instance, the player can switch options by simply paying the difference of their initial fees. On the other hand, for an investment instance, the player always has to pay the entire initial fee of the new option. We show $\sup \left\{\tilde{c}(\boldsymbol{r}, \boldsymbol{b}) \mid(\boldsymbol{r}, \boldsymbol{b}) \in I_{A}(k)\right\}=2$ and $\inf \left\{\tilde{c}(\boldsymbol{r}, \boldsymbol{b}) \mid(\boldsymbol{r}, \boldsymbol{b}) \in I_{I}(k)\right\}=2$ for any $k \geq 2$. That is to say, the best possible competitive ratio of the hardest additive instance coincides with that of the easiest investment instance.

\subsection{Related Work}

We first mention studies on the deterministic model. The classical (i.e., 2-slope) ski-rental problem was first introduced by Karlin et al. [KMRS88] in the context of snoopy caching. The problem admits an optimal 2-competitive strategy. Various practical applications can be found in the paper by Karlin [Kar96], such as context switching and virtual circuit management. Irani et al. [ISG03] discussed the multislope ski-rental problem as Dynamic Power Management. Augustine et al. [AIS08] developed an algorithm that for a given instance, outputs the best possible strategy and its competitive ratio. (While a strategy that performs the best for arbitrary instances is usually said to be optimal, in this paper we say one that performs the best for a fixed instance to be best possible.) Bejerano et al. [BCN00] provided a 4-competitive strategy for an arbitrary instance. Although their strategy was originally targeted at investment instances, 


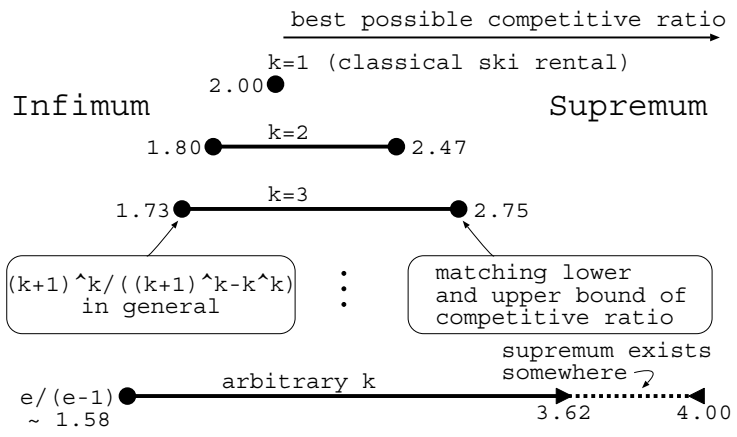

Figure 1: Illustration of the range of the best possible competitive ratio for the $(k+$ 1)-slope ski-rental problem. The infimum (supremum) is achieved by the easiest (hardest) instance, respectively.

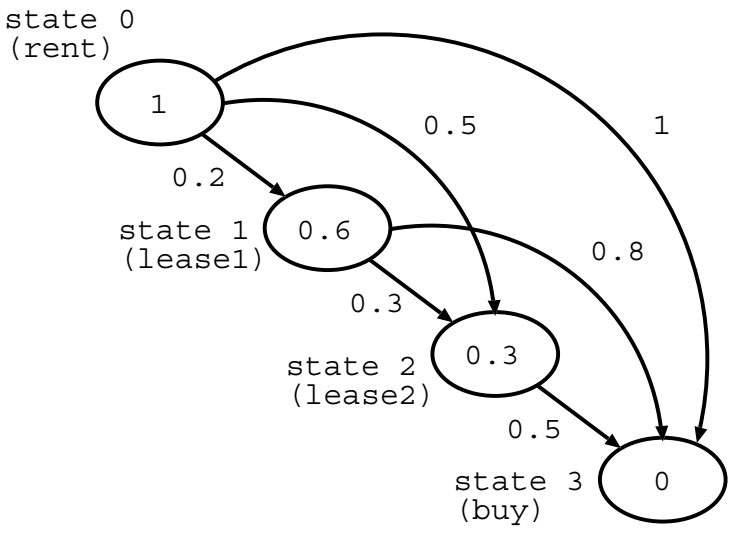

Figure 2: Example of an instance of the 4-slope ski-rental problem, $\left(r_{0}, r_{1}, r_{2}, r_{3}\right)=(1,0.6,0.3,0)$ and $\left(b_{0,1}, b_{0,2}, b_{0,3}, b_{1,2}, b_{1,3}, b_{2,3}\right)=$ $(0.2,0.5,1,0.3,0.8,0.5)$, illustrated as a state transition diagram. The value on each node and that beside each edge are the per-time cost and the transition cost, respectively.

the competitiveness straightforwardly applies to general instances as well. Damaschke [Dam03] gave a lower bound of $(5+\sqrt{5}) / 2(\approx 3.62)$ on the competitive ratio. Irani et al. [ISG03] presented a 2-competitive strategy for an arbitrary additive instance. The Bahncard problem introduced by Fleischer [Fle01] is another extension of the classical ski-rental problem.

Karlin et al. [KMMO94] provided an optimal randomized strategy for the classical ski-rental problem with a competitive ratio of $\frac{e}{e-1}$. Karlin et al. [KKR01] applied this to several problems including TCP Acknowledgment. El-Yaniv et al. [EYKL99] studied the problem in a market with interest rates. Fleischer [Fle01] gave an optimal randomized strategy for a 2-slope problem with rent and lease options. For the multislope ski-rental problem, Lotker et al. [LPSR08] presented an $e$-competitive randomized strategy for an arbitrary instance. Besides, they gave an $\frac{e}{e-1}$-competitive randomized strategy for an arbitrary additive instance, and also an algorithm that computes the best possible randomized strategy for a given additive instance.

Note. Throughout this paper, when we give a numerical value with finite precision, the rounding is always done to the nearest value.

\section{Problem Statement and Preliminaries}

\subsection{Instance}

For integer $k \geq 2$, an instance of the $(k+1)$-slope ski-rental problem consists of a pair of two vectors $(\boldsymbol{r}, \boldsymbol{b})$ that specifies $k+1$ states which we called options in Section 1. A state is referred to as a slope in some literature. We use the terminology of the multislope ski-rental problem for representing the $(k+1)$-slope ski-rental problem for all $k$ collectively.

State 0 and state $k$ stand for to rent a ski set and to buy one, respectively. States $1, \ldots, k-1$ correspond to lease a ski set in which the player pays both of some per-time fee and some initial fee. More specifically, the entries $r_{i}$ and $b_{i, j}$ are the per-time cost of state $i$ and the transition 
cost from state $i$ to state $j$, respectively. Since we are going to assume that the player starts from state 0 and may transition to another state immediately, $b_{0, j}$ is thought of as the cost for starting from state $j$ as well. We add that if one formally sets $k=1$ then one has the 2slope ski-rental problem, i.e., with no lease states, which is equivalent to the classical ski-rental problem.

We define the whole set of instances of the $(k+1)$-slope ski-rental problem, denoted by $I(k)$, to be the whole set of $(\boldsymbol{r}, \boldsymbol{b})$ such that:

$$
\begin{gathered}
1=r_{0}>r_{1}>\cdots>r_{k}=0, \\
0=b_{0,0} \leq b_{0,1} \leq \cdots \leq b_{0, k}=1, \\
b_{l, j}-b_{l, i} \leq b_{i, j} \leq b_{l, j} \text { for } 0 \leq l<i<j \leq k, \\
b_{0, j+1}\left(-r_{j-1}+r_{j}\right)+b_{0, j}\left(r_{j-1}-r_{j+1}\right)+b_{0, j-1}\left(-r_{j}+r_{j+1}\right) \leq 0 \text { for } 1 \leq j \leq k-1 .
\end{gathered}
$$

The inequalities (1) and (2) normalize the per-time and transition costs so that they are all scaled down to between zero and one. Note that in competitive analysis, the absolute magnitude of the cost itself does not matter. This normalization may look somewhat strange, but it makes sense; the number of times of skiing will also be scaled soon. Indeed, this normalization is done so that when a fractional number of times of skiing is allowed, the cost of renting a ski set for "one time" coincides with that of buying one.

The inequalities (1) and (2) say also that the sequence $\left\{r_{i}\right\}$ is decreasing and the sequence $\left\{b_{0, i}\right\}$ is non-decreasing. Consequently, per-time costs in an instance are different from each other. The left inequality in (3) is the constraint that a direct transition from state $l$ to $j$ is equal to or cheaper than a transition via another state $i$. The right inequality in (3) says that a transition from state $i$ to $j$ is not more expensive than one from state $l<i$. Refer to the paper by Augustine et al. [AIS08] for the original motivation of these restrictions in the context of Dynamic Power Management. The condition (4) guarantees that there is no redundant state to consider. The reason why the inequality in (4) is valid for this shall be clarified after the introduction of cost functions in Section 2.2.

We define two subclasses of instances which will play a significant role in the later analysis. An additive instance is such that $b_{i, j}=b_{i, l}+b_{l, j}$ holds for all $0 \leq i<l<j \leq k$. We write the whole set of additive instances with $k+1$ states as $I_{A}(k)$. An investment instance is such that $b_{i, j}=b_{0, j}$ is satisfied for all $0 \leq i<j \leq k$. Namely, the transition cost to a state is identical regardless of from which state the transition is made. The whole set of investment instances with $k+1$ states is denoted by $I_{I}(k)$.

\subsection{Strategy and Cost Functions}

We hereafter assume the number of times of skiing $t$ is a nonnegative real number. The purpose for this is simply the ease of calculation. Since the cost is piecewise continuous with respect to $t$ as we will see below, one can always interpret results in our model also in terms of the discrete model by rounding within a reasonable precision.

We mostly identify $t$ with the time, in the sense that by time $t$, the player keeps going skiing with some fixed frequency. A per-time cost is then thought of as a cost charged per unit time. At each time instant, the player who now stays at state $i$ either transitions to a different state $j(>i)$ by paying $b_{i, j}$, or keeps staying at state $i$.

A deterministic strategy of the player is a vector $\boldsymbol{x}$ with $k+1$ entries. Each entry $x_{i}$ stands for the time when the player transitions to state $i$. The sequence $\left\{x_{i}\right\}$ is assumed to be nondecreasing, since due to the constraints of $I(k)$, we consider only such instances for which the player cannot save cost by a backward transition. Without loss of generality, we assume $x_{0}=0$, 
i.e., the player always starts from state 0 ; since the player can transition anytime, a strategy of starting from state $j$ can be described by setting $x_{j}=0$. The whole set of strategies is thus written as $S=\left\{\boldsymbol{x} \mid 0=x_{0} \leq x_{1} \leq \cdots \leq x_{k}\right\}$.

For each $0 \leq i \leq k-1$, if the player adopting strategy $\boldsymbol{x}$ transitions from state $i$ to $j(>i)$, then we define a relation of $i \prec_{\boldsymbol{x}} j$. One should note that the player may transition from state $i$ directly to $j(\geq i+2)$ by skipping the states between. For such a case, we define the transition times for states $i+1, i+2, \ldots, j-1$ as $x_{i+1}=x_{i+2}=\cdots=x_{j-1}=x_{j}$. The subscript of $\prec_{\boldsymbol{x}}$ is mostly omitted when it is clear from the context.

Consider a time $t$ with $x_{i}<t<x_{i+1}$. By the time $t$, the player with strategy $\boldsymbol{x}$ will have paid a cost of

$$
O N(\boldsymbol{x}, t):=r_{i}\left(t-x_{i}\right)+\sum_{l=0}^{i-1} r_{l}\left(x_{l+1}-x_{l}\right)+\sum_{0 \leq l \leq m \leq i \text { s.t. } l \prec m} b_{l, m} .
$$

The first, second, and third terms correspond to the accumulated per-time cost after the transition to state $i$, that before the transition to state $i$, and the sum of transition costs so far, respectively.

For $t$ being exactly $x_{i}$, which is one of the transition times of strategy $\boldsymbol{x}$, we define the function as the cost immediately after the transition:

$$
O N\left(\boldsymbol{x}, x_{i}\right):=\sum_{l=0}^{i-1} r_{l}\left(x_{l+1}-x_{l}\right)+\sum_{0 \leq l \leq m \leq i \text { s.t. } l \prec m} b_{l, m} .
$$

The optimal offline player behaves optimally with $t$ known. It is observed that the optimal strategy is to transition to some state at time 0 and then keep staying there. The cost is

$$
O P T(t):=\min _{0 \leq j \leq k} O F F_{j}(t),
$$

where $O F F_{j}(t):=r_{j} t+b_{0, j}$ represents the cost of staying at state $j$ from time 0 to $t$ plus the transition cost of starting from state $j$. Let us regard $O F F_{j}(t)$ for each $j$ as a function with respect to $t$. Then, $O P T(t)$ can be seen as the lower envelope of the collection of functions $O F F_{0}(t), O F F_{1}(t), \ldots, O F F_{k}(t)$.

We are ready to explain the condition (4) for $I(k)$. This condition means that for every $j(0 \leq j \leq k)$, there exists a $t \geq 0$ such that $O F F_{j}(t)=O P T(t)$. In other words, there is no redundant state that the optimal offline player never uses. Or, one can consider that such redundant states have already been dropped from the instance. More specifically, the inequality in (4) is derived by simplifying the inequality $O F F_{j}\left(t^{*}\right) \leq O F F_{j-1}\left(t^{*}\right)$, where $t^{*}$ is the root of the linear equation $O F F_{j-1}(t)=O F F_{j+1}(t)$.

A simple necessary condition to (4) is

$$
b_{0, j} \leq 1-r_{j}, \quad \text { for } 1 \leq j \leq k-1,
$$

which will be applied in later proofs. This is confirmed as follows: One can immediately see that (5) is equivalent to $O F F_{j}(1) \leq 1$ for $1 \leq j \leq k-1$. Since $\min _{0 \leq j \leq k} O F F_{j}(1) \leq O F F_{0}(1)=$ 1 , if $O F F_{j}(1)>1$ for some $j$ then the line $O F F_{j}(t)$ can never be a part of $O P T(t)$.

\subsection{Competitiveness}

For an instance $(\boldsymbol{r}, \boldsymbol{b})$, a strategy $\boldsymbol{x}$ is said to be $c$-competitive if

$$
O N(\boldsymbol{x}, t)-c \cdot O P T(t) \leq 0
$$




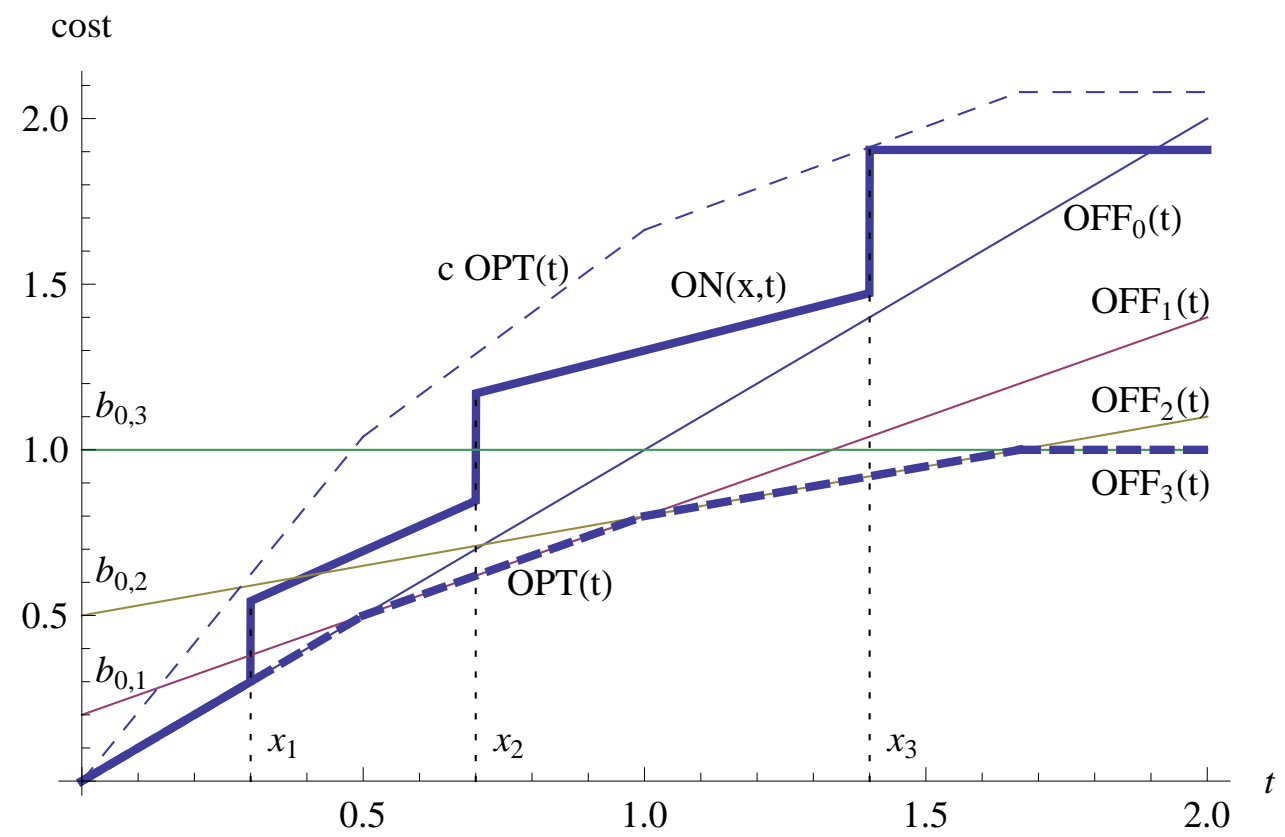

Figure 3: Cost functions for the instance of the 4-slope ski-rental problem: $\left(r_{0}, r_{1}, r_{2}, r_{3}\right)=$ $(1,0.6,0.3,0)$ and $\left(b_{0,1}, b_{0,2}, b_{0,3}, b_{1,2}, b_{1,3}, b_{2,3}\right)=(0.2,0.5,1,0.3,0.8,0.5)$. The strategy is $\left(x_{0}, x_{1}, x_{2}, x_{3}\right)=(0,0.3,0.7,1.4)$, which is approximately 1.90 -competitive.

for all $t \geq 0$. We call $c$ the competitive ratio.

We further define the best possible competitive ratio for instance $(\boldsymbol{r}, \boldsymbol{b})$ :

$$
\tilde{c}(\boldsymbol{r}, \boldsymbol{b}):=\inf \{c \mid(\exists \boldsymbol{x} \in S)(\forall t \geq 0) O N(\boldsymbol{x}, t)-c \cdot O P T(t) \leq 0\} .
$$

That is to say, $\tilde{c}(\boldsymbol{r}, \boldsymbol{b})$ is the competitive ratio of the best possible strategy for $(\boldsymbol{r}, \boldsymbol{b})$. The condition for competitiveness can be weaken by the following two arguments. The first is a lemma that allows us to concentrate on strategies satisfying $O N(\boldsymbol{x}, t)=c \cdot O P T(t)$ at every transition time. Such a strategy is called eager in the paper by Augustine et al [AIS08].

Lemma 1 ([AIS08]). Suppose that a strategy $\boldsymbol{x}$ is c-competitive. Then, there is a c-competitive strategy $\boldsymbol{x}^{\prime}$ such that $O N\left(\boldsymbol{x}^{\prime}, x_{i}^{\prime}\right)=c \cdot O P T\left(x_{i}^{\prime}\right)$ holds for all $1 \leq i \leq k$.

The second argument is on the treatment of $O P T(t)$. The minimum operation can be eliminated by imposing $k+1$ constraints instead. Hence, we will hereafter employ

$$
\tilde{c}(\boldsymbol{r}, \boldsymbol{b})=\inf \left\{c \mid(\exists \boldsymbol{x} \in S)(1 \leq \forall i \leq k, 0 \leq \forall j \leq k) O N\left(\boldsymbol{x}, x_{i}\right)-c \cdot O F F_{j}\left(x_{i}\right) \leq 0\right\} .
$$

The next proposition enables us to calculate the value of $\tilde{c}(\boldsymbol{r}, \boldsymbol{b})$ within arbitrary precision. However, the analytical relation between $(\boldsymbol{r}, \boldsymbol{b})$ and $\tilde{c}(\boldsymbol{r}, \boldsymbol{b})$ has not been known. In subsequent sections we analyze $\inf \tilde{c}(\boldsymbol{r}, \boldsymbol{b})$ and $\sup \tilde{c}(\boldsymbol{r}, \boldsymbol{b})$, revealing the easiest and the hardest instance.

Proposition 1 ([AIS08]). For any $\varepsilon>0$, there exists an algorithm that for given $(\boldsymbol{r}, \boldsymbol{b})$, computes a $(\tilde{c}(\boldsymbol{r}, \boldsymbol{b})+\varepsilon)$-competitive strategy in $O\left(k^{2} \log k \log (1 / \varepsilon)\right)$ time.

\subsection{An Example}

Here we present the example of a ski store with four options in Section 1 as an instance in $I(3)$. Although the space of scaled instances may seem narrow, one can map any instance in the real 
world to one in $I(k)$ without losing its essence. In fact, that instance is now described as $\boldsymbol{r}=$ $\left(r_{0}, r_{1}, r_{2}, r_{3}\right)=(1,0.6,0.3,0)$ and $\boldsymbol{b}=\left(b_{0,1}, b_{0,2}, b_{0,3}, b_{1,2}, b_{1,3}, b_{2,3}\right)=(0.2,0.5,1,0.3,0.8,0.5)$. Here we defined the transition costs as $b_{1,2}=b_{0,2}-b_{0,1}, b_{1,3}=b_{0,3}-b_{0,1}$, and $b_{2,3}=b_{0,3}-b_{0,2}$, which were not specified in Section 1 . The resulting instance thus belongs to $I_{A}(3)$. Figure 2 shows this instance as a state transition diagram.

Figure 3 illustrates cost functions for this instance and the strategy $\boldsymbol{x}=\left(x_{0}, x_{1}, x_{2}, x_{3}\right)=$ $(0,0.3,0.7,1.4)$. The graph of $O N(\boldsymbol{x}, t)$ jumps at $t=x_{1}, x_{2}$, and $x_{3}$ due to state transitions. Elsewhere it increases linearly with slope $r_{i}$. The graph of $O P T(t)$ shows the cost when the player ideally transitions to an optimal state at the beginning.

The competitive ratio of strategy $\boldsymbol{x}$ is $c=\frac{175}{92} \approx 1.90$. This is easily confirmed; $O N(\boldsymbol{x}, t)-$ $c \cdot O P T(t)$ is zero for $t=1.4$ and negative elsewhere. We remark that strategy $\boldsymbol{x}$ is not the best possible for $(\boldsymbol{r}, \boldsymbol{b})$. The best possible strategy for this instance is calculated as $\overline{\boldsymbol{x}}=$ $\left(\bar{x}_{0}, \bar{x}_{1}, \bar{x}_{2}, \bar{x}_{3}\right) \approx(0,0.26,0.54,1.67)$, whose competitive ratio is approximately 1.77 . Hence, we have that $\tilde{c}(\boldsymbol{r}, \boldsymbol{b}) \approx 1.77$ for this $(\boldsymbol{r}, \boldsymbol{b})$. Competitiveness can be explained visually in Figure 3 : If the graph of $O N(\boldsymbol{x}, t)$ is drawn below that of $c \cdot O P T(t)$, then strategy $\boldsymbol{x}$ is $c$-competitive.

\section{Infimum of the Best Possible Competitive Ratio}

\subsection{Fixed $k$}

Intuitively, the infimum of $\tilde{c}(\boldsymbol{r}, \boldsymbol{b})$ indicates to what extent the strategy can be improved when one chooses the easiest instance for the player. We derive the infimum by solving a mathematical program in which the strategy and instance are both formulated as variables. Note that such a formulation is possible because the infimum is written as

$$
\begin{aligned}
& \inf \{\tilde{c}(\boldsymbol{r}, \boldsymbol{b}) \mid(\boldsymbol{r}, \boldsymbol{b}) \in I(k)\} \\
& =\inf \left\{c \mid(\exists(\boldsymbol{r}, \boldsymbol{b}) \in I(k))(\exists \boldsymbol{x} \in S)(1 \leq \forall i \leq k, 0 \leq \forall j \leq k) O N\left(\boldsymbol{x}, x_{i}\right)-c \cdot O F F_{j}\left(x_{i}\right) \leq 0\right\} .
\end{aligned}
$$

We first see that we can narrow down the space of instances to consider. The simple lemma below implies that there is an instance in $I_{A}(k)$ which achieves an infimum.

Lemma 2. Let $(\boldsymbol{x}, \boldsymbol{r}, \boldsymbol{b}, c)$ be such that $\boldsymbol{x} \in S,(\boldsymbol{r}, \boldsymbol{b}) \in I(k)$, and $O N\left(\boldsymbol{x}, x_{i}\right)-c \cdot O F F_{j}\left(x_{i}\right) \leq 0$ for all $1 \leq i \leq k$ and $0 \leq j \leq k$. Set $\boldsymbol{b}^{\prime}$ as $b_{i, j}^{\prime}:=b_{0, j}-b_{0, i}$ for $0<i<j \leq k$, and $b_{0, i}^{\prime}:=b_{0, i}$ for $0 \leq i \leq k$. Then, also for $\left(\boldsymbol{r}, \boldsymbol{b}^{\prime}\right) \in I_{A}(k), O N\left(\boldsymbol{x}, x_{i}\right)-c \cdot O F F_{j}\left(x_{i}\right) \leq 0$ holds for all $1 \leq i \leq k$ and $0 \leq j \leq k$.

Proof. The fact that $\left(\boldsymbol{r}, \boldsymbol{b}^{\prime}\right) \in I_{A}(k)$ directly follows from the definition of additive instance. Fix $i(1 \leq i \leq k)$ and $j(0 \leq j \leq i)$ arbitrarily. For all $l \prec m \leq i, b_{l, m} \geq b_{0, m}-b_{0, l}=b_{l, m}^{\prime}$ holds. Hence, by replacing $\boldsymbol{b}$ with $\boldsymbol{b}^{\prime}$, the value of $O N\left(\boldsymbol{x}, x_{i}\right)$ decreases, or remains the same. On the other hand, the value of $O F F_{j}\left(\boldsymbol{x}, x_{i}\right)$ does not change. Consequently, we have for $\left(\boldsymbol{r}, \boldsymbol{b}^{\prime}\right)$, $O N\left(\boldsymbol{x}, x_{i}\right)-c \cdot O F F_{j}\left(x_{i}\right) \leq 0$.

We use the notation

$$
g_{i, j}(\boldsymbol{x}, \boldsymbol{r}, \boldsymbol{b}, c):=O N\left(\boldsymbol{x}, x_{i}\right)-c \cdot O F F_{j}\left(x_{i}\right)=\sum_{l=0}^{i-1} r_{l}\left(x_{l+1}-x_{l}\right)+b_{0, i}-c\left(r_{j} x_{i}+b_{0, j}\right) .
$$

for describing the condition for strategy $\boldsymbol{x}$ to be $c$-competitive. Note that for an additive instance, the sum of transition costs equals simply $b_{0, i}$. We are ready to formulate a mathematical 
program.

$(\mathcal{P})$ minimize $c$

$$
\begin{array}{cl}
\text { subject to } & g_{i, j}(\boldsymbol{x}, \boldsymbol{r}, \boldsymbol{b}, c) \leq 0, \quad \text { for } 1 \leq i \leq k, 0 \leq j \leq k, \\
\boldsymbol{x} \in S,(\boldsymbol{r}, \boldsymbol{b}) \in I_{A}(k) . &
\end{array}
$$

In spite of its nonconvexity, we will solve the program analytically and obtain an explicit solution. The infimum is indeed a minimum. Postponing the proof of optimality, we here present the solution.

Theorem 1. The following $(\overline{\boldsymbol{x}}, \overline{\boldsymbol{r}}, \overline{\boldsymbol{b}}, \bar{c})$ is a global optimum to $(\mathcal{P})$ :

$$
\begin{array}{rlrl}
\bar{x}_{i} & =\frac{i}{k} & & \text { for } 0 \leq i \leq k, \\
\bar{r}_{i} & =\bar{c}+(1-\bar{c})\left(1+\frac{1}{k}\right)^{i} & & \text { for } 0 \leq i \leq k, \\
\bar{b}_{0, i} & =1-\bar{r}_{i} & & \text { for } 0 \leq i \leq k, \\
\bar{b}_{i, j} & =\bar{b}_{0, j}-\bar{b}_{0, i} & & \text { for } 0<i<j \leq k, \\
\bar{c} & =\frac{(k+1)^{k}}{(k+1)^{k}-k^{k}} . &
\end{array}
$$

Corollary 1. It holds $\inf \{\tilde{c}(\boldsymbol{r}, \boldsymbol{b}) \mid(\boldsymbol{r}, \boldsymbol{b}) \in I(k)\}=\min \{\tilde{c}(\boldsymbol{r}, \boldsymbol{b}) \mid(\boldsymbol{r}, \boldsymbol{b}) \in I(k)\}=\frac{(k+1)^{k}}{(k+1)^{k}-k^{k}}$. That is to say, for any instance of the $(k+1)$-slope ski-rental problem, no strategy achieves a competitive ratio below $\frac{(k+1)^{k}}{(k+1)^{k}-k^{k}}$.

The solution consists of the easiest instance for the player and the best possible strategy for it. Here we give some numerical examples. For $k=2$, we have $\bar{c}=\frac{9}{5}=1.80,\left(\bar{x}_{0}, \bar{x}_{1}, \bar{x}_{2}\right)=$ $\left(0, \frac{1}{2}, 1\right),\left(\bar{r}_{0}, \bar{r}_{1}, \bar{r}_{2}\right)=\left(1, \frac{3}{5}, 0\right)$, and $\left(\bar{b}_{0,1}, \bar{b}_{0,2}, \bar{b}_{1,2}\right)=\left(\frac{2}{5}, 1, \frac{3}{5}\right)$. And for $k=3, \bar{c}=\frac{64}{37} \approx 1.73$, $\left(\bar{x}_{0}, \bar{x}_{1}, \bar{x}_{2}, \bar{x}_{3}\right)=\left(0, \frac{1}{3}, \frac{2}{3}, 1\right),\left(\bar{r}_{0}, \bar{r}_{1}, \bar{r}_{2}, \bar{r}_{3}\right)=\left(1, \frac{28}{37}, \frac{16}{37}, 0\right)$, and $\left(\bar{b}_{0,1}, \bar{b}_{0,2}, \bar{b}_{0,3}, \bar{b}_{1,2}, \bar{b}_{1,3}, \bar{b}_{2,3}\right)=$ $\left(\frac{9}{37}, \frac{21}{37}, 1, \frac{12}{37}, \frac{28}{37}, \frac{16}{37}\right)$. Figures $4,5,6$, and 7 show the easiest instances and the cost functions of the best possible strategies.

Please recall that $O P T(t)$ is the lower envelope of $O F F_{0}(t), O F F_{1}(t), \ldots, O F F_{k}(t)$. One can see that in Figures 5 and 7 , every $O F F_{j}(t)$ with $1 \leq j \leq k-1$ degenerates to a single point. This means that for these instances, the offline player has only the choice of state 0 or $k$. To use other states cannot contribute to cost-saving. On the other hand, the resulting best possible strategy keeps transitioning to the next state at equal time intervals while exploiting all the states available.

Our original interest was how much the online player takes advantage of the easiness of the instance. However, the result is a bit different from what we expected: The resulting instance is unfavorable to the offline player, rather than easy to the online player. This is seen as, in a sense, a limit of worst-case competitive analysis.

\subsection{Arbitrary $k$}

We have

$$
\bar{c}=\frac{(k+1)^{k}}{(k+1)^{k}-k^{k}}=1+\frac{1}{\left(1+\frac{1}{k}\right)^{k}-1} \rightarrow 1+\frac{1}{e-1}=\frac{e}{e-1}
$$

as $k \rightarrow \infty$, which provides the following corollary in a straightforward manner. The best possible competitive ratio can never be improved down to one. The corollary is more suggestive 


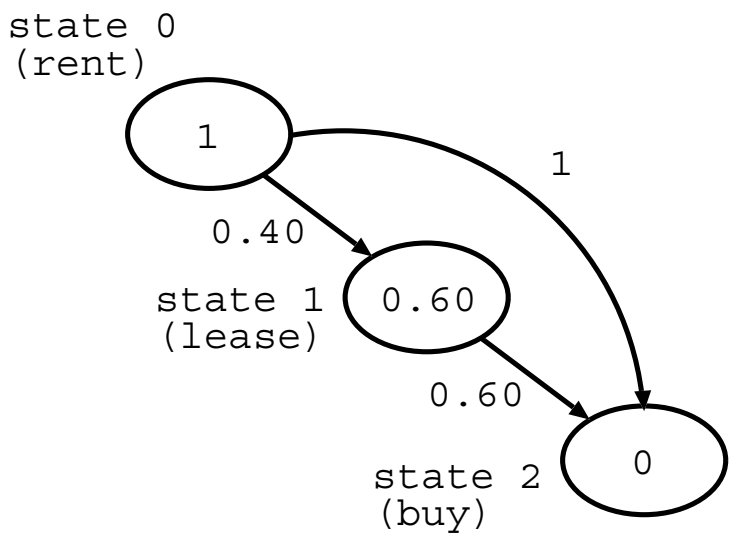

Figure 4: Easiest instance of the 3-slope skirental problem: $\left(\bar{r}_{0}, \bar{r}_{1}, \bar{r}_{2}\right)=\left(1, \frac{3}{5}, 0\right)$, and $\left(\bar{b}_{0,1}, \bar{b}_{0,2}, \bar{b}_{1,2}\right)=\left(\frac{2}{5}, 1, \frac{3}{5}\right)$.

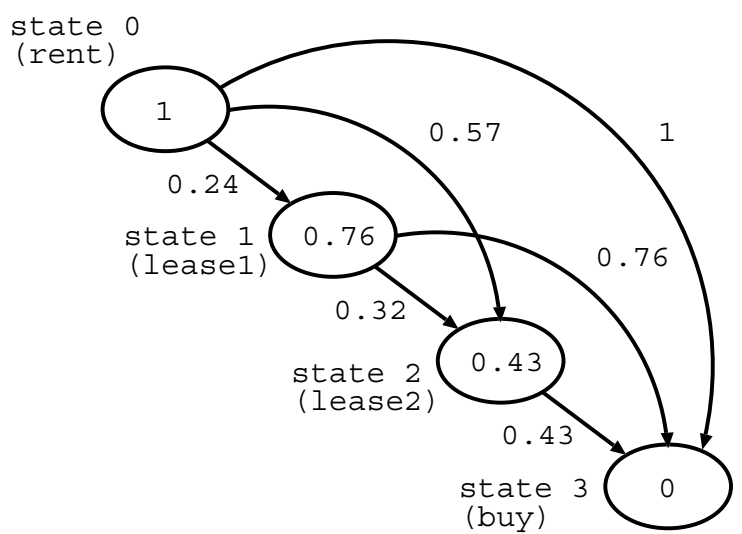

Figure 6: Easiest instance of the 4-slope ski-rental problem: $\left(\bar{r}_{0}, \bar{r}_{1}, \bar{r}_{2}, \bar{r}_{3}\right)=\left(1, \frac{28}{37}, \frac{16}{37}, 0\right)$, and $\left(\bar{b}_{0,1}, \bar{b}_{0,2}, \bar{b}_{0,3}, \bar{b}_{1,2}, \bar{b}_{1,3}, \bar{b}_{2,3}\right)=$ $\left(\frac{9}{37}, \frac{21}{37}, 1, \frac{12}{37}, \frac{28}{37}, \frac{16}{37}\right)$.

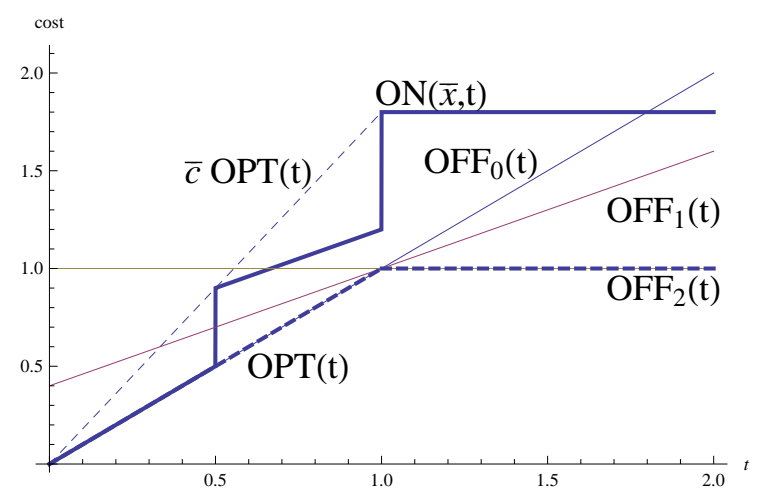

Figure 5: Cost functions for the easiest instance of the 3 -slope ski-rental problem. The best possible strategy is $\left(\bar{x}_{0}, \bar{x}_{1}, \bar{x}_{2}\right)=$ $\left(0, \frac{1}{2}, 1\right)$.

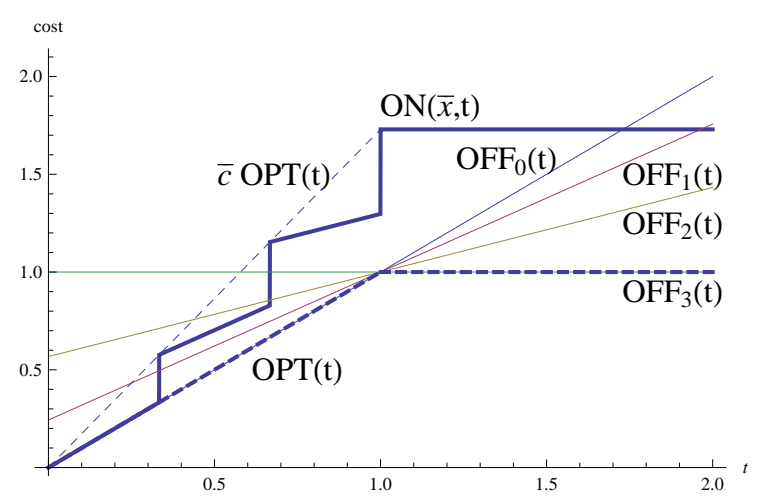

Figure 7: Cost functions for the easiest instance of the 4-slope ski-rental problem. The best possible strategy is $\left(\bar{x}_{0}, \bar{x}_{1}, \bar{x}_{2}, \bar{x}_{3}\right)=$ $\left(0, \frac{1}{3}, \frac{2}{3}, 1\right)$. 
in the context of Dynamic Power Management: Even if arbitrarily many energy-saving states are implemented, there is a limit of improvement in competitiveness.

Corollary 2. It holds $\inf \{\tilde{c}(\boldsymbol{r}, \boldsymbol{b}) \mid(\boldsymbol{r}, \boldsymbol{b}) \in I(k), k \geq 2\}=\frac{e}{e-1} \approx 1.58$. That is to say, for any instance of the multislope ski-rental problem, no strategy achieves a competitive ratio below $\frac{e}{e-1}$.

The value of the obtained infimum coincides with the competitive ratio of the optimal randomized strategy for the classical (i.e., 2-slope) ski-rental problem [KMMO94]. This fact is explained as follows: Fix finite $k \geq 2$. For the classical ski-rental problem, consider a randomized strategy that buys a ski set at time $\frac{i}{k}$ with a probability of $p_{i}$ for each $1 \leq i \leq k$. Let $\operatorname{EON}(t)$ be its expected cost incurred by time $t$. Choose $\left\{p_{i}\right\}$ so as to minimize $\inf _{t \geq 0} \frac{E O N(t)}{O P T(t)}$, that is, the competitive ratio of the randomized strategy. Then, we can observe that $\operatorname{EON}(t)=O N(\overline{\boldsymbol{x}}, t)$ for all $t \geq 0$, where $\overline{\boldsymbol{x}}$ is the strategy given in Theorem 1 . As $k \rightarrow \infty$, the player becomes allowed to buy a ski set at arbitrary timing and therefore the competitive ratio $\inf _{t \geq 0} \frac{E O N(t)}{O P T(t)}$ approaches the optimal value $\frac{e}{e-1}$. Accordingly, $\inf \{\tilde{c}(\boldsymbol{r}, \boldsymbol{b}) \mid(\boldsymbol{r}, \boldsymbol{b}) \in I(k)\} \rightarrow \frac{e}{e-1}$ as well.

\subsection{Proof of Theorem 1}

The proof is sketched as follows: We first consider a parametric optimization problem $\left(\mathcal{Q}_{r^{*}}\right)$ of $(\mathcal{P})$, and guess a solution to it. Although problem $\left(\mathcal{Q}_{r^{*}}\right)$ is nonconvex, the guessed solution is proved to be indeed optimal. Then, we formulate a problem $(\mathcal{R})$ whose objective function is the optimal value function to $\left(\mathcal{Q}_{\boldsymbol{r}^{*}}\right)$. By solving problem $(\mathcal{R})$, we finally obtain a global optimum to $(\mathcal{P})$.

First, by regarding $\boldsymbol{r}$ in program $(\mathcal{P})$ as a parameter, denoted by $\boldsymbol{r}^{*}$, we obtain a parametric optimization problem $\left(\mathcal{Q}_{r^{*}}\right)$.

$$
\begin{aligned}
\left(\mathcal{Q}_{\boldsymbol{r}^{*}}\right) \quad \text { minimize } c & \\
\text { subject to } & g_{i, j}\left(\boldsymbol{x}, \boldsymbol{r}^{*}, \boldsymbol{b}, c\right) \leq 0, \\
& \boldsymbol{x} \in S,\left(\boldsymbol{r}^{*}, \boldsymbol{b}\right) \in I_{A}(k) .
\end{aligned}
$$

For every $c$, denote by $M(c)$ the set of $(\boldsymbol{x}, \boldsymbol{b})$ such that $(\boldsymbol{x}, \boldsymbol{b}, c)$ is feasible to $\left(\mathcal{Q}_{\boldsymbol{r}^{*}}\right)$. We can rewrite problem $\left(\mathcal{Q}_{\boldsymbol{r}^{*}}\right)$ as

$$
\text { minimize } c \text { subject to } M(c) \neq \emptyset \text {. }
$$

Here we give a lemma that provides a sufficient condition of optimality for general problems of this form. We remark that this lemma will further be applied to the analysis of $\sup \tilde{c}(\boldsymbol{r}, \boldsymbol{b})$ in Section 4.

Lemma 3. Let $M(c)$ be any set labeled by a real number $c$. Suppose that for any $c<c^{\prime}$, $M(c) \subsetneq M\left(c^{\prime}\right)$ holds, i.e., $M(c)$ is a proper subset of $M\left(c^{\prime}\right)$. If there exists $\bar{c}$ such that $M(\bar{c})$ is a singleton, i.e., a set consisting of a single element, then $\inf \{c \mid M(c) \neq \emptyset\}=\min \{c \mid M(c) \neq$ $\emptyset\}=\bar{c}$.

Proof. Let $A:=\{c \mid M(c) \neq \emptyset\}$. Trivially, $\bar{c} \in A$. For every $c>\bar{c}$, we have $M(\bar{c}) \subsetneq M(c)$ by assumption. Then $M(c) \neq \emptyset$ and therefore $c \in A$. On the other hand, for every $c<\bar{c}$, $M(c) \subsetneq M(\bar{c})$. Since $M(\bar{c})$ is a singleton, $M(c)=\emptyset$. That is to say, $c \notin A$.

Therefore, for any $c \in A, \bar{c} \leq c$ holds. Together with $\bar{c} \in A$, we conclude $\bar{c}=\min A$. 
Through computer experiments for some small values of $k$, we guess $\left(\boldsymbol{x}^{*}, \boldsymbol{b}^{*}, c^{*}\right)$ that satisfies the following equation system to be optimal:

$$
\begin{aligned}
b_{0, i} & =1-r_{i}^{*} & & \text { for } 1 \leq i \leq k, \\
g_{i, 0}\left(\boldsymbol{x}, \boldsymbol{r}^{*}, \boldsymbol{b}, c\right) & =0 & & \text { for } 1 \leq i \leq k, \\
g_{k, k}\left(\boldsymbol{x}, \boldsymbol{r}^{*}, \boldsymbol{b}, c\right) & =0 . & &
\end{aligned}
$$

Let us solve this equation system. Recalling (6), we see (14) with $i=1$ and $c=1$ :

$$
r_{0}^{*}\left(x_{1}-x_{0}\right)+b_{0, i}-\left(r_{0}^{*} x_{1}+b_{0,0}\right)=x_{1}+\left(1-r_{1}^{*}\right)-x_{1}=1-r_{1}^{*}=0,
$$

which does not satisfy (1). Therefore, we can assume $c \neq 1$. By taking the difference of (14) with plugging (13), we have for each $1 \leq i \leq k$,

$$
\begin{aligned}
0 & =g_{i+1,0}\left(\boldsymbol{x}, \boldsymbol{r}^{*}, \boldsymbol{b}, c\right)-g_{i, 0}\left(\boldsymbol{x}, \boldsymbol{r}^{*}, \boldsymbol{b}, c\right) \\
& =r_{i}^{*}\left(x_{i+1}-x_{i}\right)+\left(\left(1-r_{i+1}^{*}\right)-\left(1-r_{i}^{*}\right)\right)-c\left(x_{i+1}-x_{i}\right) \\
& =-\left(x_{i+1}-x_{i}\right)\left(c-r_{i}^{*}\right)+r_{i}^{*}-r_{i+1}^{*} .
\end{aligned}
$$

We thus have

$$
x_{i+1}-x_{i}=\frac{r_{i}^{*}-r_{i+1}^{*}}{c-r_{i}^{*}} .
$$

From (14) with $i=1$ and $x_{0}=0$, it is derived that

$$
x_{1}=\frac{1-r_{1}^{*}}{c-1} .
$$

This implies also that for the feasibility of $\boldsymbol{x}, c>1$ is required. By repeatedly applying (16), we get, in general for $1 \leq i \leq k$,

$$
x_{i}=\sum_{l=0}^{i-1} \frac{r_{l}^{*}-r_{l+1}^{*}}{c-r_{l}^{*}} .
$$

Also,

$$
0=g_{k, k}\left(\boldsymbol{x}, \boldsymbol{r}^{*}, \boldsymbol{b}, c\right)-g_{k, 0}\left(\boldsymbol{x}, \boldsymbol{r}^{*}, \boldsymbol{b}, c\right)=-c+c x_{k}
$$

implies $x_{k}=1$. From (17) with $i=k$, we know that $c$ should be a root of

$$
h\left(\boldsymbol{r}^{*}, c\right):=\sum_{i=0}^{k-1} \frac{r_{i}^{*}-r_{i+1}^{*}}{c-r_{i}^{*}}-1=0 .
$$

Lemma 4. See $h\left(\boldsymbol{r}^{*}, c\right)=0$ as an equation with respect to $c$ under the assumption of $c>1$. The equation has a unique real root between 1 and 2.

Proof. By considering just the first term in the summation, we obtain

$$
h\left(\boldsymbol{r}^{*}, c\right)>\frac{1-r_{1}^{*}}{c-1}-1 .
$$

Recall that $r_{1}^{*}<1$. We know by $(19)$ that $h\left(\boldsymbol{r}^{*}, c\right)>0$ for any $c \in\left(1,2-r_{1}^{*}\right)$. We also have

$$
h\left(\boldsymbol{r}^{*}, 2\right)=\sum_{i=0}^{k-1} \frac{r_{i}^{*}-r_{i+1}^{*}}{2-r_{i}^{*}}-1<\sum_{i=0}^{k-1} \frac{r_{i}^{*}-r_{i+1}^{*}}{2-1}-1=r_{0}^{*}-r_{k}^{*}-1=0 .
$$


Furthermore, for $1<c$,

$$
\frac{\partial h}{\partial c}=-\sum_{i=0}^{k-1} \frac{r_{i}^{*}-r_{i+1}^{*}}{\left(c-r_{i}^{*}\right)^{2}}<0
$$

implies that $h\left(\boldsymbol{r}^{*}, c\right)$ decreases monotonically. These facts guarantee unique existence of a real root between 1 and 2 .

Denote by $c^{*}$ the root of $h\left(\boldsymbol{r}^{*}, c\right)=0$ greater than one. Then, using (17), we determine $x_{1}^{*}, \ldots, x_{k}^{*}$. Set $x_{0}^{*}=0$ and $b_{0, i}^{*}=1-r_{i}^{*}$ for $1 \leq i \leq k$. Next, our guess turns out to be correct.

Lemma 5. It follows that $\left(\boldsymbol{x}^{*}, \boldsymbol{b}^{*}, c^{*}\right)$ is a global optimum to problem $\left(\mathcal{Q}_{\boldsymbol{r}^{*}}\right)$.

Proof. First, we confirm the feasibility of $\left(\boldsymbol{x}^{*}, \boldsymbol{b}^{*}, c^{*}\right)$. It is easy to see that $\boldsymbol{x}^{*} \in S$ and $\left(\boldsymbol{r}^{*}, \boldsymbol{b}^{*}\right) \in$ $I_{A}(k)$. Needless to say, $\boldsymbol{x}^{*}$ and $c^{*}$ satisfy the equation system of (13), (14), and (15). We check other constraints. By (13) and $x_{i}^{*} \leq x_{k}^{*}=1$ for $1 \leq i \leq k-1$, we have $g_{i, j}\left(\boldsymbol{x}^{*}, \boldsymbol{r}^{*}, \boldsymbol{b}^{*}, c^{*}\right) \leq$ $g_{i, 0}\left(\boldsymbol{x}^{*}, \boldsymbol{r}^{*}, \boldsymbol{b}^{*}, c^{*}\right)=0$ for $1 \leq i \leq k-1$ and $1 \leq j \leq k$. We also have $g_{k, j}\left(\boldsymbol{x}^{*}, \boldsymbol{r}^{*}, \boldsymbol{b}^{*}, c^{*}\right)=$ $g_{k, k}\left(\boldsymbol{x}^{*}, \boldsymbol{r}^{*}, \boldsymbol{b}^{*}, c^{*}\right)=0$ for $1 \leq j \leq k-1$. The feasibility of $\left(\boldsymbol{x}^{*}, \boldsymbol{b}^{*}, c^{*}\right)$ is thus confirmed. The rest of the proof is to show its optimality by applying Lemma 3 .

(I) Recall the problem in the form of (12). We first prove that for any $c<c^{\prime}, M(c) \subsetneq M\left(c^{\prime}\right)$ holds. $M(c) \subseteq M\left(c^{\prime}\right)$ is immediately proved, since for any $c<c^{\prime}$ and any feasible $(\boldsymbol{x}, \boldsymbol{b}, c)$, we have for all $1 \leq i \leq k$ and $0 \leq j \leq k$,

$$
\sum_{l=0}^{i-1} r_{l}^{*}\left(x_{l+1}-x_{l}\right)+b_{0, i} \leq c\left(r_{j}^{*} x_{i}+b_{0, j}\right)<c^{\prime}\left(r_{j}^{*} x_{i}+b_{0, j}\right) .
$$

Lemma 1 implies that if $\left(\boldsymbol{x}, \boldsymbol{b}, c^{\prime}\right)$ is feasible, then there exists $\left(\boldsymbol{x}^{\prime}, \boldsymbol{b}, c^{\prime}\right)$ such that for each $1 \leq i \leq k, g_{i, j}\left(\boldsymbol{x}^{\prime}, \boldsymbol{r}^{*}, \boldsymbol{b}, c^{\prime}\right)=0$ holds for some $0 \leq j \leq k$. For such pairs of $i$ and $j$, and any $c<c^{\prime}$

$$
\sum_{l=0}^{i-1} r_{l}^{*}\left(x_{l+1}^{\prime}-x_{l}^{\prime}\right)+b_{0, i}=c^{\prime}\left(r_{j}^{*} x_{i}^{\prime}+b_{0, j}\right)>c\left(r_{j}^{*} x_{i}^{\prime}+b_{0, j}\right),
$$

which claims that $\left(\boldsymbol{x}^{\prime}, \boldsymbol{b}, c\right)$ is not in $M\left(c^{\prime}\right)$. Thus, $M(c) \subsetneq M\left(c^{\prime}\right)$.

(II) We next prove that $M\left(c^{*}\right)$ is a singleton $\left\{\left(\boldsymbol{x}^{*}, \boldsymbol{b}^{*}\right)\right\}$. Observe that for each $c, M(c)$ is a polyhedron since with fixed $r^{*}$ and $c$, the constraints are all linear. Therefore, $M(c)$ is a connected set for fixed $c$. Hence, it suffices to show that any neighbor of $\left(\boldsymbol{x}^{*}, \boldsymbol{b}^{*}\right)$, except itself, is not contained in $M\left(c^{*}\right)$. Let $(\boldsymbol{x}, \boldsymbol{b})$ be a point that is arbitrarily chosen from the neighborhood of $\left(\boldsymbol{x}^{*}, \boldsymbol{b}^{*}\right)$. In the following we see that $(\boldsymbol{x}, \boldsymbol{b})$ violates at least one of the constraints.

The next inequalities are obtained by differentiating $g_{i, j}$.

$$
\begin{array}{rlrl}
\left.\frac{\partial g_{i, 0}}{\partial x_{i}}\right|_{\left(\boldsymbol{x}^{*}, \boldsymbol{b}^{*}, c^{*}\right)} & =r_{i-1}^{*}-c^{*} r_{0}^{*}<0, & & \text { for } 1 \leq i \leq k, \\
\left.\frac{\partial g_{k, 0}}{\partial x_{i}}\right|_{\left(\boldsymbol{x}^{*}, \boldsymbol{b}^{*}, c^{*}\right)} & =r_{i-1}^{*}-r_{i}^{*}>0, & & \text { for } 1 \leq i \leq k-1, \\
\left.\frac{\partial g_{k, k}}{\partial x_{k}}\right|_{\left(\boldsymbol{x}^{*}, \boldsymbol{b}^{*}, c^{*}\right)} & =r_{k-1}^{*}-c^{*} r_{k}^{*}>0 . &
\end{array}
$$

From these inequalities we have that: For $1 \leq i \leq k-1$, if $x_{i}>x_{i}^{*}$ then $g_{k, 0}$ becomes positive. Otherwise, $g_{i, 0}$ becomes positive. If $x_{k}>x_{k}^{*}$ then $g_{k, k}$ becomes positive. Otherwise, $g_{k, 0}$ becomes positive. 
As for $\boldsymbol{b}$, we first note that $g_{k, j}\left(\boldsymbol{x}^{*}, \boldsymbol{r}^{*}, \boldsymbol{b}^{*}, c^{*}\right)=g_{k, k}\left(\boldsymbol{x}^{*}, \boldsymbol{r}^{*}, \boldsymbol{b}^{*}, c^{*}\right)=0$ holds for all $0 \leq j \leq$ $k-1$. Then,

$$
\left.\frac{\partial g_{k, j}}{\partial b_{0, j}}\right|_{\left(\boldsymbol{x}^{*}, \boldsymbol{b}^{*}, c^{*}\right)}=-c^{*}<0
$$

implies that if $b_{0, j}<b_{0, j}^{*}$ then the point is infeasible. Next, recall (13). If $b_{0, j}>b_{0, j}^{*}$, we have $b_{0, j}>1-r_{j}^{*}$. Then, the condition (5) is not satisfied, which means $\left(\boldsymbol{r}^{*}, \boldsymbol{b}\right) \notin I(k)$. The entries $b_{i, j}(0<i<j \leq k)$ need not be considered, since we now concentrate on additive instances.

Consequently, Lemma 3 states that $\left(\boldsymbol{x}^{*}, \boldsymbol{b}^{*}, c^{*}\right)$ is a global optimum to problem $\left(\mathcal{Q}_{\boldsymbol{r}^{*}}\right)$.

Finally, we regard $c^{*}$ as the optimal value function to $\left(\mathcal{Q}_{r^{*}}\right)$ and formulate a problem $(\mathcal{R})$ with $\boldsymbol{r}$ being a variable. Lemma 6 gives a solution to $(\mathcal{R})$.

$$
\begin{aligned}
& (\mathcal{R}) \quad \operatorname{minimize} c^{*} \\
& \text { subject to } h\left(\boldsymbol{r}, c^{*}\right)=0,1<c^{*}<2,1=r_{0}>r_{1}>\cdots>r_{k}=0 .
\end{aligned}
$$

Lemma 6. It follows that $\overline{\boldsymbol{r}}$ in (8) is a global optimum to problem $(\mathcal{R})$. The optimal value is $\bar{c}$ in (11).

Proof. We derive

$$
\begin{aligned}
h\left(\boldsymbol{r}, c^{*}\right) & =\sum_{i=0}^{k-1} \frac{r_{i}-r_{i+1}}{c^{*}-r_{i}}-1 \\
& =\sum_{i=0}^{k-1}\left(\frac{c^{*}-r_{i+1}}{c^{*}-r_{i}}-1\right)-1 \\
& =\sum_{i=0}^{k-1} \frac{c^{*}-r_{i+1}}{c^{*}-r_{i}}-k-1 \\
& \geq k\left(\prod_{i=0}^{k-1} \frac{c^{*}-r_{i+1}}{c^{*}-r_{i}}\right)^{\frac{1}{k}}-k-1 \\
& =k\left(\frac{c^{*}-r_{1}}{c^{*}-r_{0}} \cdot \frac{c^{*}-r_{2}}{c^{*}-r_{1}} \cdots \cdot \frac{c^{*}-r_{k}}{c^{*}-r_{k-1}}\right)^{\frac{1}{k}}-k-1 \\
& =k\left(\frac{c^{*}-r_{k}}{c^{*}-r_{0}}\right)^{\frac{1}{k}}-k-1 \\
& =k\left(\frac{c^{*}}{c^{*}-1}\right)^{\frac{1}{k}}-k-1 .
\end{aligned}
$$

The inequality is known as the inequality of arithmetic and geometric means. Note that this is applicable since $c^{*}-r_{i}$ is positive for all $i$. The equality holds when

$$
\frac{c^{*}-r_{1}}{c^{*}-r_{0}}=\frac{c^{*}-r_{2}}{c^{*}-r_{1}}=\cdots=\frac{c^{*}-r_{k}}{c^{*}-r_{k-1}} .
$$

If one solves the equation

$$
k\left(\frac{c^{*}}{c^{*}-1}\right)^{\frac{1}{k}}-k-1=0
$$


then one obtains its unique root

$$
c^{*}=\frac{(k+1)^{k}}{(k+1)^{k}-k^{k}} \text {. }
$$

It is easily seen that the function

$$
c^{*} \mapsto\left(\frac{c^{*}}{c^{*}-1}\right)^{\frac{1}{k}}=\left(1+\frac{1}{c^{*}-1}\right)^{\frac{1}{k}}
$$

decreases monotonically. Since $h\left(\boldsymbol{r}, c^{*}\right)=0$, we know by $(20)$ that $c^{*}$ cannot be smaller than $\frac{(k+1)^{k}}{(k+1)^{k}-k^{k}}$. In the rest of the proof we will show that $c^{*}$ can indeed take this minimum value, by providing $r_{0}, \ldots, r_{k}$ that fulfill (21), (22), and $1=r_{0}>r_{1}>\cdots>r_{k}=0$.

We solve the equations in (21) with respect to $r_{0}, \ldots, r_{k}$. Let

$$
\frac{c^{*}-r_{1}}{c^{*}-r_{0}}=\frac{c^{*}-r_{2}}{c^{*}-r_{1}}=\cdots=\frac{c^{*}-r_{k}}{c^{*}-r_{k-1}}=\gamma \text {. }
$$

Then we have

$$
0=h\left(\boldsymbol{r}, c^{*}\right)=\sum_{i=0}^{k-1} \frac{c^{*}-r_{i+1}}{c^{*}-r_{i}}-k-1=\gamma k-k-1 .
$$

Therefore, we get $\gamma=1+\frac{1}{k}$. If we fix $r_{0}=1$, the sequence $\left\{r_{i}\right\}$ is recursively determined as

$$
r_{i}=c^{*}+\left(1-c^{*}\right)\left(1+\frac{1}{k}\right)^{i}
$$

for $1 \leq i \leq k$. We then derive

$$
r_{k}=c^{*}+\left(1-c^{*}\right)\left(1+\frac{1}{k}\right)^{k}=\frac{(k+1)^{k}}{(k+1)^{k}-k^{k}}-\frac{k^{k}}{(k+1)^{k}-k^{k}} \cdot \frac{(k+1)^{k}}{k^{k}}=0 .
$$

It also follows that for $0 \leq i \leq k-1$,

$r_{i+1}-r_{i}=\left(c^{*}-r_{i}\right)-\left(c^{*}-r_{i+1}\right)=\left(c^{*}-r_{i}\right)-\gamma\left(c^{*}-r_{i}\right)=(1-\gamma)\left(c^{*}-r_{i}\right)=-\frac{1}{k}\left(c^{*}-r_{i}\right)<0$.

The obtained sequence $\left\{r_{i}\right\}$ is thus consistent with $1=r_{0}>r_{1}>\cdots>r_{k}=0$.

We obtain $\overline{\boldsymbol{x}}$ and $\overline{\boldsymbol{b}}$ in (7), (9), and (10) by plugging $\overline{\boldsymbol{r}}$ into $\boldsymbol{x}^{*}$ and $\boldsymbol{b}^{*}$. Lemmas 5 and 6 state that $(\overline{\boldsymbol{x}}, \overline{\boldsymbol{r}}, \overline{\boldsymbol{b}}, \bar{c})$ is a global optimum to $(\mathcal{P})$.

\section{Supremum of the Best Possible Competitive Ratio}

We first see that $\sup \tilde{c}(\boldsymbol{r}, \boldsymbol{b})$ is equal to the matching upper and lower bound on the competitive ratio in the ordinary sense. In the literature on the multislope ski-rental problem, $c_{u}$ is said to be an upper bound if for all $(\boldsymbol{r}, \boldsymbol{b})$, there exists a strategy $\boldsymbol{x}$ which is $c_{u}$-competitive. It is observed that the set of such $c_{u}$ is equivalent to the set of upper bounds of $\tilde{c}(\boldsymbol{r}, \boldsymbol{b})$ over arbitrary instances. Hence, if one identifies $\sup \tilde{c}(\boldsymbol{r}, \boldsymbol{b})$, it is equal to the least upper bound. On the other hand, $c_{l}$ is called a lower bound if there exists $(\boldsymbol{r}, \boldsymbol{b})$ for which any strategy $\boldsymbol{x}$ has a competitive ratio of at least $c_{l}$. Analogously, we have that $\sup \tilde{c}(\boldsymbol{r}, \boldsymbol{b})$ coincides with the greatest lower bound. We add that $c_{l} \leq \sup \tilde{c}(\boldsymbol{r}, \boldsymbol{b}) \leq c_{u}$ holds accordingly.

To seek the infimum, it is sufficient to consider only additive instances, as we discussed in Section 3. In a similar way the following lemma guarantees that an investment instance achieves a supremum. In the rest of this section, we deal with only investment instances. For the sake of simpler notation, for $(\boldsymbol{r}, \boldsymbol{b}) \in I_{I}(k)$, we will denote $b_{i, j}$ with $0 \leq i \leq j-1$ simply by $b_{j}$. 
Lemma 7. Given $(\boldsymbol{r}, \boldsymbol{b}) \in I(k)$, set $b_{i, j}^{\prime}=b_{0, j}$ for all $0 \leq i<j \leq k$, and $b_{0,0}^{\prime}=0$. Suppose that for $\left(\boldsymbol{r}, \boldsymbol{b}^{\prime}\right) \in I_{I}(k)$ and $\boldsymbol{x} \in S, O N\left(\boldsymbol{x}, x_{i}\right)-c \cdot O F F_{j}\left(x_{i}\right) \leq 0$ holds for all $1 \leq i \leq k$ and $0 \leq j \leq k$. Then, also for $(\boldsymbol{r}, \boldsymbol{b}), O N\left(\boldsymbol{x}, x_{i}\right)-c \cdot O F F_{j}\left(x_{i}\right) \leq 0$ for all $1 \leq i \leq k$ and $0 \leq j \leq k$.

Proof. From the condition (3), we know $b_{i, j}^{\prime}=b_{0, j} \geq b_{i, j}$ for all $0 \leq i<j \leq k$. The lemma immediately follows since for each $1 \leq i \leq k$, the value of $O N\left(\boldsymbol{x}, x_{i}\right)$ for $\left(\boldsymbol{r}, \boldsymbol{b}^{\prime}\right)$ is at least that for $(\boldsymbol{r}, \boldsymbol{b})$.

\subsection{Fixed $k$}

The analysis of the supremum is much more involved than that of the infimum, since one must consider minimization with respect to strategy $\boldsymbol{x}$ and maximization with respect to instance $(\boldsymbol{r}, \boldsymbol{b})$. We here present a supremum each for $k=2$ and $k=3$. Refer to Sections 4.3 and 4.4 for the proofs. The resulting instance is the hardest instance for the player in the sense that for that instance the player can never achieve a better competitive ratio than the value of the supremum. Figures 8, 9, 10, and 11 illustrate the hardest instances and the cost functions of the best possible strategies. As discussed at the beginning of this section, our suprema establish a matching upper and lower bound on the competitive ratio each for the 3-slope and 4-slope ski-rental problems in the ordinary sense.

Theorem 2. It holds that $\sup \{\tilde{c}(\boldsymbol{r}, \boldsymbol{b}) \mid(\boldsymbol{r}, \boldsymbol{b}) \in I(2)\}=\bar{c}$, where $\bar{c}$ is the unique real root of $c^{3}-4 c^{2}+5 c-3=0$, approximately 2.47. The instance that achieves the supremum is $\left(\bar{r}_{0}, \bar{r}_{1}, \bar{r}_{2}\right)=\left(1, \varepsilon_{1}, 0\right)$ for $\varepsilon_{1} \rightarrow 0$ and $\left(\bar{b}_{1}, \bar{b}_{2}\right) \approx(0.68,1)$, where $\bar{b}_{1}$ is the unique real root of $b_{1}^{3}+b_{1}-1=0$. The best possible strategies are $\left(\bar{x}_{0}, \bar{x}_{1}, \bar{x}_{2}\right)=\left(0, \bar{b}_{1}, \bar{b}_{1}\right) \approx(0,0.68,0.68)$ and $\left(\bar{x}_{0}, \bar{x}_{1}, \bar{x}_{2}\right) \rightarrow\left(0, \frac{1}{2}\left(\sqrt{4 \bar{b}_{1}+1}-1\right), \infty\right) \approx(0,0.47, \infty)$.

Corollary 3. Let $\bar{c}$ be the unique real root of $c^{3}-4 c^{2}+5 c-3=0$, which is approximately 2.47. For any instance of the 3-slope ski-rental problem, there exists a strategy which has a competitive ratio of $\bar{c}$. Moreover, there exists an instance of the 3-slope ski-rental problem for which any strategy has a competitive ratio of at least $\bar{c}$.

Theorem 3. It holds that $\sup \{\tilde{c}(\boldsymbol{r}, \boldsymbol{b}) \mid(\boldsymbol{r}, \boldsymbol{b}) \in I(3)\}=\overline{\boldsymbol{c}}$, where $\overline{\boldsymbol{c}}$ is the unique real root of $c^{3}$ $5 c^{2}+8 c-5=0$, approximately 2.75 . The instance that achieves the supremum is $\left(\bar{r}_{0}, \bar{r}_{1}, \bar{r}_{2}, \bar{r}_{3}\right)=$ $\left(1, \varepsilon_{1}, \varepsilon_{2}, 0\right)$ for $\varepsilon_{1} \rightarrow 0$ and $\varepsilon_{2} \rightarrow 0$ with $\varepsilon_{1}>\varepsilon_{2}$, and $\left(\bar{b}_{1}, \bar{b}_{2}, \bar{b}_{3}\right) \approx(0.40,0.70,1)$, where $\bar{b}_{1}=$ $\frac{\bar{c}^{2}-3 \bar{c}+3}{\bar{c}^{2}-\bar{c}+1}$ and $\bar{b}_{2}=\frac{\bar{c}^{2}-2 \bar{c}+2}{\bar{c}^{2}-\bar{c}+1}$. The best possible strategies are $\left(\bar{x}_{0}, \bar{x}_{1}, \bar{x}_{2}, \bar{x}_{3}\right) \rightarrow\left(0, \frac{\bar{b}_{1}}{\bar{c}-1}, \infty, \infty\right) \approx$ $(0,0.23, \infty, \infty)$ and $\left(\bar{x}_{0}, \bar{x}_{1}, \bar{x}_{2}, \bar{x}_{3}\right) \rightarrow\left(0, \frac{\bar{b}_{2}}{\bar{c}-1}, \frac{\bar{b}_{2}}{\bar{c}-1}, \infty\right) \approx(0,0.40,0.40, \infty)$.

Corollary 4. Let $\bar{c}$ be the unique real root of $c^{3}-5 c^{2}+8 c-5=0$, which is approximately 2.75. For any instance of the 4-slope ski-rental problem, there exists a strategy which has a competitive ratio of $\bar{c}$. Moreover, there exists an instance of the 4-slope ski-rental problem for which any strategy has a competitive ratio of at least $\bar{c}$.

\subsection{Arbitrary $k$}

It seems difficult to extend our analysis on fixed $k$ to arbitrary $k$ straightforwardly. We merely know the interval where the supremum value lies, implied by the existing upper and lower bounds on the competitive ratio in the standard sense. There still remains a gap between $(5+\sqrt{5}) / 2 \approx 3.62$ and 4 .

Theorem 4 ([Dam03]). It holds $\sup \{\tilde{c}(\boldsymbol{r}, \boldsymbol{b}) \mid(\boldsymbol{r}, \boldsymbol{b}) \in I(k), k \geq 2\} \geq(5+\sqrt{5}) / 2 \approx 3.62$.

Theorem 5 ([BCN00]). It holds $\sup \{\tilde{c}(\boldsymbol{r}, \boldsymbol{b}) \mid(\boldsymbol{r}, \boldsymbol{b}) \in I(k), k \geq 2\} \leq 4$. 


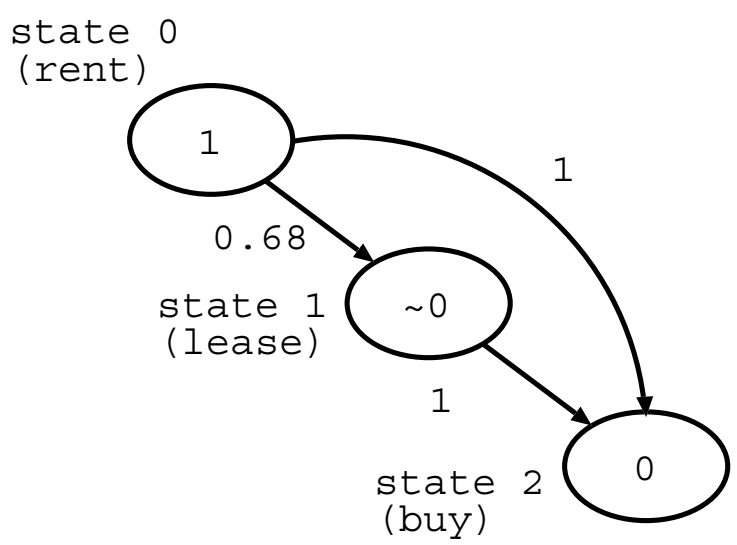

Figure 8: Hardest instance of the 3-slope skirental problem: $\left(\bar{r}_{0}, \bar{r}_{1}, \bar{r}_{2}\right)=\left(1, \varepsilon_{1}, 0\right)$ and $\left(\bar{b}_{1}, \bar{b}_{2}\right)=(0.68,1)$ with $\varepsilon_{1} \rightarrow 0$.

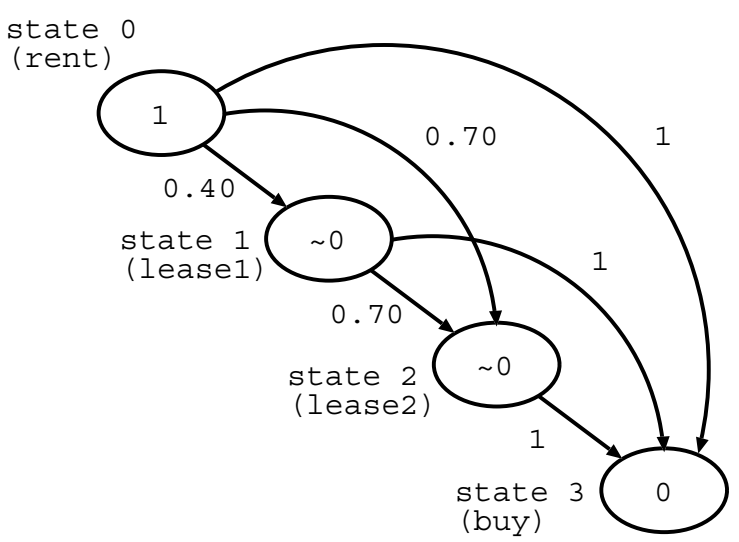

Figure 10: Hardest instance of the 4slope ski-rental problem: $\left(\bar{r}_{0}, \bar{r}_{1}, \bar{r}_{2}, \bar{r}_{3}\right)=$ $\left(1, \varepsilon_{1}, \varepsilon_{2}, 0\right)$ and $\left(\bar{b}_{1}, \bar{b}_{2}, \bar{b}_{3}\right)=(0.40,0.70,1)$ with $\varepsilon_{1} \rightarrow 0$ and $\varepsilon_{2} \rightarrow 0$.

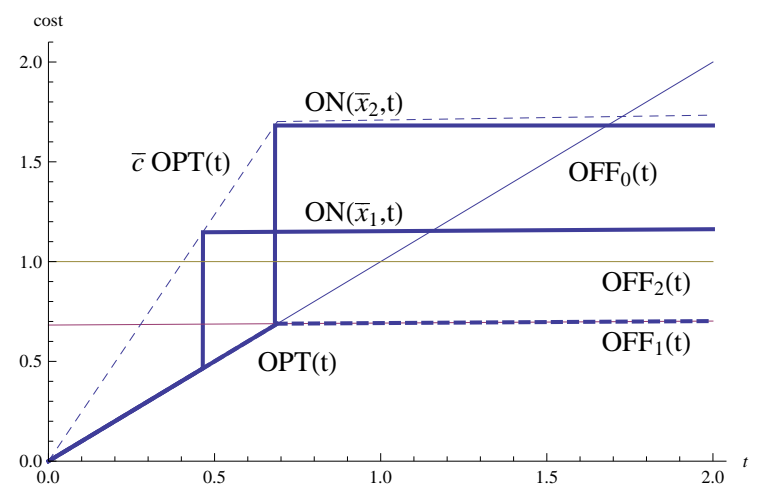

Figure 9: Cost functions for the hardest instance of the 3-slope ski-rental problem, where $\bar{r}_{1}$ is set 0.01 . The best possible strategies are $\left(\bar{x}_{0}, \bar{x}_{1}, \bar{x}_{2}\right)=(0,0.68,0.68)$ and $(0,0.47, \infty)$.

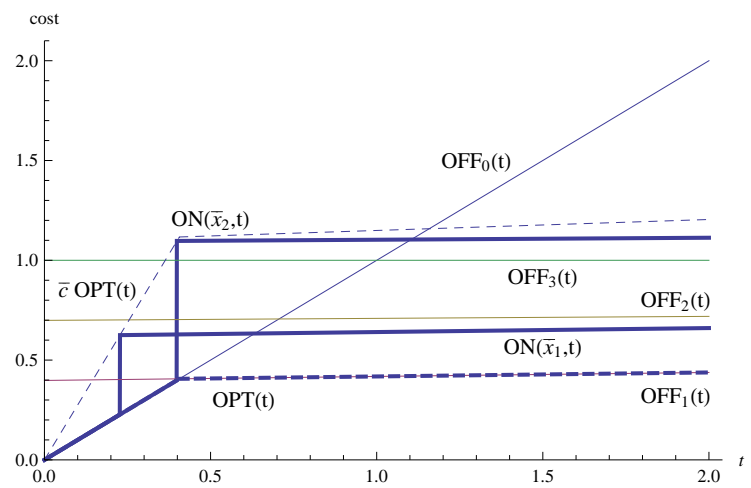

Figure 11: Cost functions for the hardest instance of the 4-slope ski-rental problem, where $\bar{r}_{1}$ and $\bar{r}_{2}$ are set 0.02 and 0.01 , respectively. The best possible strategies are $\left(\bar{x}_{0}, \bar{x}_{1}, \bar{x}_{2}, \bar{x}_{3}\right)=(0,0.23, \infty, \infty)$ and $(0,0.40,0.40, \infty)$. 


\subsection{Proof of Theorem 2}

The theorem is proved by solving a problem of the form "maximize $\min \{\cdots\}$ ". Although some subproblems which appear in this proof can be solved by simple calculus, we insist on the technique based on the Lemma 3 for further application to larger $k$. Lemma 7 claims that it suffices to consider only instances in $I_{I}(2)$.

(I) States 0, 1, and 2 are now available for the player. Naturally, the player has two choices: (a) $0 \prec 2$ (i.e., skipping state 1 ) and (b) $0 \prec 1 \prec 2$ (i.e., with no skip). In what follows we will refer to these as types of strategy. One should note that unlike the case of an additive instance, here we must consider the constraint of competitiveness separately, depending on the type of strategy. We thus denote $O N\left(\boldsymbol{x}, x_{i}\right)-c \cdot O F F_{j}\left(x_{i}\right)$ by $g_{i, j}^{(a)}(\boldsymbol{x}, \boldsymbol{r}, \boldsymbol{b}, c)$ for $\boldsymbol{x}$ of type (a) and by $g_{i, j}^{(b)}(\boldsymbol{x}, \boldsymbol{r}, \boldsymbol{b}, c)$ for $\boldsymbol{x}$ of type (b). We omit the arguments of $g$ when they are clear.

We are now going to describe the best possible competitive ratio $\tilde{c}(\boldsymbol{r}, \boldsymbol{b})$ in terms of $r_{i}$ 's and $b_{j}$ 's. For this aim, for a given instance $(\boldsymbol{r}, \boldsymbol{b}) \in I_{I}(2)$, we first need to get a strategy that is the best possible among those of each type. This is done by solving the following problems: For type (a),

minimize $c$

$$
\begin{aligned}
& \text { subject to } g_{2,0}^{(a)}=x_{2}+1-c x_{2} \leq 0, \\
& g_{2,1}^{(a)}=x_{2}+1-c\left(r_{1} x_{2}+b_{1}\right) \leq 0, \\
& g_{2,2}^{(a)}=x_{2}+1-c \leq 0, \\
& 0<x_{2} .
\end{aligned}
$$

Note that for type (a), it holds that $g_{1, j}^{(a)}=g_{2, j}^{(a)}$ for $0 \leq j \leq 2$. For type (b),

minimize $c$

$$
\begin{aligned}
& \text { subject to } g_{1,0}^{(b)}=x_{1}+b_{1}-c x_{1} \leq 0, \\
& g_{1,1}^{(b)}=x_{1}+b_{1}-c\left(r_{1} x_{1}+b_{1}\right) \leq 0, \\
& g_{1,2}^{(b)}=x_{1}+b_{1}-c \leq 0, \\
& g_{2,0}^{(b)}=x_{1}+r_{1}\left(x_{2}-x_{1}\right)+b_{1}+1-c x_{2} \leq 0, \\
& g_{2,1}^{(b)}=x_{1}+r_{1}\left(x_{2}-x_{1}\right)+b_{1}+1-c\left(r_{1} x_{2}+b_{1}\right) \leq 0, \\
& g_{2,2}^{(b)}=x_{1}+r_{1}\left(x_{2}-x_{1}\right)+b_{1}+1-c \leq 0, \\
& 0<x_{1}<x_{2} .
\end{aligned}
$$

We solve these problems by applying Lemma 3. We begin with type (a). For every $c$, denote by $X^{(a)}(c)$ the set of feasible $\boldsymbol{x}$, that is, $\left\{\boldsymbol{x} \mid(0 \leq \forall j \leq 2) g_{2, j}^{(a)} \leq 0,0<x_{2}\right\}$. From the following discussion we can see that $X^{(a)}(c) \subsetneq X^{(a)}\left(c^{\prime}\right)$ for any $c<c^{\prime}$ : By definition of $g_{i, j}^{(a)}$, clearly $X^{(a)}(c) \subseteq X^{(a)}\left(c^{\prime}\right)$ holds for any $c<c^{\prime}$. Lemma 1 implies that if $\left(\boldsymbol{x}, c^{\prime}\right)$ is feasible, then there exists $\left(\boldsymbol{x}^{\prime}, c^{\prime}\right)$ such that $g_{2, j}^{(a)}\left(\boldsymbol{x}^{\prime}, \boldsymbol{r}, \boldsymbol{b}, c^{\prime}\right)=0$ holds for some $0 \leq j \leq 2$. Since $g_{2, j}^{(a)}\left(\boldsymbol{x}^{\prime}, \boldsymbol{r}, \boldsymbol{b}, c\right)>$ $g_{2, j}^{(a)}\left(\boldsymbol{x}^{\prime}, \boldsymbol{r}, \boldsymbol{b}, c^{\prime}\right)$ for $c<c^{\prime}, X^{(a)}(c)$ does not contain $\boldsymbol{x}^{\prime}$. Thus, $X^{(a)}(c) \subsetneq X^{(a)}\left(c^{\prime}\right)$.

By Lemma 3, if we find $c$ such that $X^{(a)}(c)$ is a singleton $\{\boldsymbol{x}\}$ then $\boldsymbol{x}$ is a solution. As a result of the following argument we will conclude: If $r_{1}<b_{1}, \hat{\boldsymbol{x}}=\left(0, \frac{b_{1}}{1-r_{1}}, \frac{b_{1}}{1-r_{1}}\right)$ and $\hat{c}=1+\frac{1-r_{1}}{b_{1}}$ are a solution, which is a root of $g_{2,0}^{(a)}=g_{2,1}^{(a)}=0$. Otherwise, $\hat{\boldsymbol{x}}=\left(0, \frac{1-b_{1}}{r_{1}}, \frac{1-b_{1}}{r_{1}}\right)$ and $\hat{c}=1+\frac{1-b_{1}}{r_{1}}$, which is a root of $g_{2,1}^{(a)}=g_{2,2}^{(a)}=0$. 
Let us check the case $r_{1}<b_{1}$. The solution presented above is feasible; indeed, $g_{2,2}^{(a)}=$ $\hat{x}_{2}+1-\hat{c}=\frac{b_{1}}{1-r_{1}}-\frac{1-r_{1}}{b_{1}} \leq 0$ since $b_{1} \leq 1-r_{1}$. The inequalities

$$
\begin{aligned}
& \left.\frac{\partial g_{2,0}^{(a)}}{\partial x_{2}}\right|_{(\hat{\boldsymbol{x}}, \hat{c})}=1-\hat{c}=-\frac{1-r_{1}}{b_{1}}<0, \\
& \left.\frac{\partial g_{2,1}^{(a)}}{\partial x_{2}}\right|_{(\hat{\boldsymbol{x}}, \hat{c})}=1-\hat{c} r_{1}=1-\left(1+\frac{1-r_{1}}{b_{1}}\right) r_{1}=\left(1-r_{1}\right)\left(1+\frac{r_{1}}{b_{1}}\right)>0
\end{aligned}
$$

imply that any neighbor of $\hat{\boldsymbol{x}}$ violates either $g_{2,0}^{(a)} \leq 0$ or $g_{2,1}^{(a)} \leq 0$. Together with connectivity of $X^{(a)}(c)$ for any fixed $c, X^{(a)}(\hat{c})$ is a singleton. Therefore $\hat{\boldsymbol{x}}=\left(0, \frac{b_{1}}{1-r_{1}}, \frac{b_{0,1}}{1-r_{1}}\right)$ is a solution when $r_{1}<b_{1}$.

For the case $r_{1}>b_{1}$, we know that the solution above is feasible; $g_{2,0}^{(a)}=\hat{x}_{2}+1-\hat{c} \hat{x}_{2}=$ $1+\frac{1-b_{1}}{r_{1}}-\left(1+\frac{1-b_{1}}{r_{1}}\right) \frac{1-b_{1}}{r_{1}}=1-\left(\frac{1-b_{1}}{r_{1}}\right)^{2} \leq 0$ since $r_{1} \leq 1-b_{1}$. We have

$$
\begin{aligned}
& \left.\frac{\partial g_{2,1}^{(a)}}{\partial x_{2}}\right|_{(\hat{\boldsymbol{x}}, \hat{c})}=1-\hat{c} r_{1}=1-\left(1+\frac{1-r_{1}}{b_{1}}\right) r_{1}=b_{1}-r_{1}<0, \\
& \left.\frac{\partial g_{2,2}^{(a)}}{\partial x_{2}}\right|_{(\hat{\boldsymbol{x}}, \hat{c})}=1>0 .
\end{aligned}
$$

As the same as the case $r_{1}<b_{1}$, these inequalities imply that $\hat{\boldsymbol{x}}=\left(0, \frac{1-b_{1}}{r_{1}}, \frac{1-b_{1}}{r_{1}}\right)$ is a solution. We add that when $r_{1}=b_{1}$, the both strategies become optimal. The problem for type (a) is thus solved.

A solution to the problem for type (b) is $\hat{\boldsymbol{x}}=\left(0, \frac{-1+\sqrt{1+4 b_{1}\left(1-r_{1}\right)}}{2\left(1-r_{1}\right)}, \frac{1-b_{1}}{r_{1}}\right)$ and $\hat{c}=\frac{1}{2}(3+$ $\left.\sqrt{1+4 b_{1}\left(1-r_{1}\right)}\right)$, for any instance in $I_{I}(2)$. This is a feasible root of $g_{1,0}^{(b)}=g_{2,1}^{(b)}=g_{2,2}^{(b)}=0$. The feasibility is checked by simply plugging the solution into other constraints. Let us see the optimality. Analogously for type (a), let $X^{(b)}(c):=\left\{\boldsymbol{x} \mid(1 \leq \forall i \leq 2,0 \leq \forall j \leq 2) g_{i, j}^{(b)} \leq 0,0<\right.$ $\left.x_{1}<x_{2}\right\}$. With the help of Lemma 3 , we guarantee the optimality by showing that $X^{(b)}(\hat{c})$ is a singleton $\{\hat{\boldsymbol{x}}\}$. Indeed, the following inequalities show that any neighbor of $\hat{\boldsymbol{x}}$ violates either $g_{1,0}^{(b)} \leq 0, g_{2,1}^{(b)} \leq 0$, or $g_{2,2}^{(b)} \leq 0$ :

$$
\begin{aligned}
& \left.\frac{\partial g_{1,0}^{(b)}}{\partial x_{1}}\right|_{(\hat{\boldsymbol{x}}, \hat{c})}=1-\hat{c}=-\frac{1}{2}-\frac{1}{2} \sqrt{1+4 b_{1}\left(1-r_{1}\right)}<0, \\
& \left.\frac{\partial g_{2,1}^{(b)}}{\partial x_{1}}\right|_{(\hat{\boldsymbol{x}}, \hat{c})}=1-r_{1}>0, \\
& \left.\frac{\partial g_{2,1}^{(b)}}{\partial x_{2}}\right|_{(\hat{\boldsymbol{x}}, \hat{c})}=r_{1}-\hat{c} r_{1}=r_{1}\left(-\frac{1}{2}-\frac{1}{2} \sqrt{1+4 b_{1}\left(1-r_{1}\right)}\right)<0, \\
& \left.\frac{\partial g_{2,2}^{(b)}}{\partial x_{2}}\right|_{(\hat{\boldsymbol{x}}, \hat{c})}=r_{1}>0 .
\end{aligned}
$$

The optimality of $\hat{\boldsymbol{x}}$ to the problem for type (b) is thus guaranteed. 
As a result, we obtain the best possible competitive ratio for $(\boldsymbol{r}, \boldsymbol{b}) \in I_{I}(2)$ in a closed form

$$
\tilde{c}(\boldsymbol{r}, \boldsymbol{b})=\min \left\{1+\frac{1-r_{1}}{b_{1}}, 1+\frac{1-b_{1}}{r_{1}}, \frac{1}{2}\left(3+\sqrt{1+4 b_{1}\left(1-r_{1}\right)}\right)\right\} .
$$

(II) We next maximize $\tilde{c}(\boldsymbol{r}, \boldsymbol{b})$ with $\boldsymbol{r}$ being a parameter $\boldsymbol{r}^{*}$. Namely, only $b_{1}$ is regarded as a variable. We formulate a parametric problem:

$$
\begin{aligned}
\operatorname{maximize} c & \\
\text { subject to } c & \leq \hat{c}_{1}:=1+\frac{1-r_{1}^{*}}{b_{1}}, \\
c & \leq \hat{c}_{2}:=1+\frac{1-b_{1}}{r_{1}^{*}}, \\
c & \leq \hat{c}_{3}:=\frac{1}{2}\left(3+\sqrt{1+4 b_{1}\left(1-r_{1}^{*}\right)}\right) .
\end{aligned}
$$

We solve this problem again with the help of Lemma 3. For every $c$, denote by $B(c)$ the feasible set of $b_{1}$. By easy calculation, we have that for $c>c^{\prime}, B(c) \subsetneq B\left(c^{\prime}\right)$. Let us find a root of the equation $c=\hat{c}_{1}=\hat{c}_{3}$. We guess the root to be a solution to the problem. Solving the equation with respect to each of $b_{1}$ and $c$, we obtain

$$
\begin{aligned}
b_{1}^{3}+b_{1}-1+r_{1}^{*} & =0, \\
c^{3}-4 c^{2}+5 c-3-r_{1}^{* 2}+2 r_{1}^{*} & =0 .
\end{aligned}
$$

Basic calculus guarantees that for any $r_{1}^{*}$, each of the equations (24) and (25) has a unique real root. We denote the roots by $b_{1}^{*}\left(r_{1}^{*}\right)$ and $c^{*}\left(r_{1}^{*}\right)$, respectively. Moreover, it is revealed that $0<b_{1}^{*}\left(r_{1}^{*}\right)<1$ and $2<c^{*}\left(r_{1}^{*}\right)<3$. We have the inequalities

$$
\begin{aligned}
& \left.\frac{\partial \hat{c}_{1}}{\partial b_{1}}\right|_{b_{1}^{*}\left(r_{1}^{*}\right)}=-\frac{1-r_{1}^{*}}{b_{1}^{*}\left(r_{1}^{*}\right)^{2}}<0, \\
& \left.\frac{\partial \hat{c}_{3}}{\partial b_{1}}\right|_{b_{1}^{*}\left(r_{1}^{*}\right)}=\frac{1-r_{1}^{*}}{\sqrt{1+4 b_{1}^{*}\left(r_{1}^{*}\right)\left(1-r_{1}^{*}\right)}}>0 .
\end{aligned}
$$

Hence, no neighbor of $b_{1}^{*}\left(r_{1}^{*}\right)$ is contained in $B\left(c^{*}\left(r_{1}^{*}\right)\right)$. Together with connectivity of $B(c)$ for any $c, B\left(c^{*}\left(r_{1}^{*}\right)\right)$ turns out to be a singleton. Therefore $b_{1}^{*}\left(r_{1}^{*}\right)$ is a solution.

(III) Our final task is to maximize $c^{*}\left(r_{1}^{*}\right)$ with regarding $r_{1}^{*}$ as a variable. By investigating the equation (25), we know that $c^{*}\left(r_{1}^{*}\right)$ increases as $r_{1}^{*}$ decreases. This means that $c^{*}\left(r_{1}^{*}\right)$ achieves a supremum as $r_{1}^{*} \rightarrow 0$. The value is the unique real root $\bar{c}$ of the equation

$$
c^{3}-4 c^{2}+5 c-3=0,
$$

which is approximately 2.47. It is also derived that as $r_{1}^{*} \rightarrow 0, b_{1}^{*}\left(r_{1}^{*}\right)$ approaches $\bar{b}_{1} \approx 0.68$, which is the unique real root of the equation

$$
b_{1}^{3}+b_{1}-1=0 .
$$

Going back to the solutions to the problems in (I), we get the best possible strategies. For type (a), since it has turned out that the supremum is achieved when $r_{1}<b_{1}$, the strategy for the case $r_{1}<b_{1}$ achieves the supremum. We have $\left(\bar{x}_{0}, \bar{x}_{1}, \bar{x}_{2}\right)=\left(0, \bar{b}_{1}, \bar{b}_{1}\right) \approx(0,0.68,0.68)$. For type (b), we have $\left(\bar{x}_{0}, \bar{x}_{1}, \bar{x}_{2}\right) \rightarrow\left(0, \frac{1}{2}\left(-1+\sqrt{4 \bar{b}_{1}+1}\right), \infty\right) \approx(0,0.47, \infty)$.

Thus, we have proved $\sup \left\{\tilde{c}(\boldsymbol{r}, \boldsymbol{b}) \mid(\boldsymbol{r}, \boldsymbol{b}) \in I_{I}(2)\right\}=\overline{\boldsymbol{c}}$. Together with Lemma 7, we conclude $\sup \{\tilde{c}(\boldsymbol{r}, \boldsymbol{b}) \mid(\boldsymbol{r}, \boldsymbol{b}) \in I(2)\}=\bar{c}$. 


\subsection{Proof of Theorem 3}

The proof is done in the same way as Theorem 2. Thus, here we give a sketch. Lemma 7 claims that it is sufficient to consider investment instances. We will omit expressions of solutions to subproblems; if we try to describe the solutions in a closed form then even one of them will require large space. Therefore, instead of writing a solution explicitly, we will give an equation system that has a root of that value.

(I) We describe the best possible competitive ratio $\tilde{c}(\boldsymbol{r}, \boldsymbol{b})$ for an arbitrarily given $(\boldsymbol{r}, \boldsymbol{b}) \in$ $I_{I}(3)$, by solving the best possible strategy belonging to each type. The types of strategy that should be considered here are: Type (a) $0 \prec 3$, type (b) $0 \prec 1 \prec 3$, type (c) $0 \prec 2 \prec 3$, and type (d) $0 \prec 1 \prec 2 \prec 3$. We will denote $g_{i, j}(\boldsymbol{x}, \boldsymbol{r}, \boldsymbol{b}, c):=O N\left(\boldsymbol{x}, x_{i}\right)-c \cdot O F F_{j}\left(x_{i}\right)$ according to these types as $g_{i, j}^{(a)}, g_{i, j}^{(b)}, g_{i, j}^{(c)}$, and $g_{i, j}^{(d)}$.

For each type, the best possible competitive ratio over strategies belonging to that type is given as a solution of the problem with $\boldsymbol{x}$ being a variable in the form

$$
\begin{aligned}
& \text { minimize } c \\
& \text { subject to } g_{i, j}^{(\cdot)} \leq 0, \quad \text { for } 1 \leq i \leq 3,0 \leq j \leq 3 .
\end{aligned}
$$

The candidate of a solution is the real roots of equation systems consisting of: For every $(l, m)$ satisfying $0 \leq l \prec m \leq 3, g_{l, j}^{(\cdot)}=0$ and $g_{m, j^{\prime}}^{(\cdot)}=0$ with some $0 \leq j \leq j^{\prime} \leq 3$. Although some system may include an irrelevant real root that never becomes the best possible, this does not matter here; we get the best possible competitive ratio by taking the minimum over all the candidates and all the types. Such a root will vanish then.

We here present three real roots of $c$ which play an important role soon. Note that each root is obtained by eliminating $\boldsymbol{x}$ from the equation system.

- (B): The real root of $g_{1,0}^{(b)}=g_{3,2}^{(b)}=g_{3,3}^{(b)}=0$.

- (C): The real root of $g_{2,0}^{(c)}=g_{2,1}^{(c)}=g_{3,2}^{(c)}=0$.

- (D): The real root of $g_{1,0}^{(d)}=g_{2,0}^{(d)}=g_{3,2}^{(d)}=g_{3,3}^{(d)}=0$.

(II) Let $r_{1}$ and $r_{2}$ be parameters. We consider the maximization of $\tilde{c}(\boldsymbol{r}, \boldsymbol{b})$ with variables $b_{1}$ and $b_{2}$. With the help of Lemma 3 , it turns out that the optimal value $c^{*}$ of that maximization problem is equal to a real root of the equations stating that the three real roots $(B),(C)$, and (D) take the same value. By eliminating $b_{1}$ and $b_{2}$, we obtain an equation that consists of only $c^{*}$, $r_{1}$, and $r_{2}$.

(III) The final step is to maximize $c^{*}$ with respect to variables $r_{1}$ and $r_{2}$. By differentiating implicitly the equation which we have got as a result of (II), we know that $c^{*}$ grows, as $r_{1}$ and $r_{2}$ decrease. We take $r_{1} \rightarrow 0$ and $r_{2} \rightarrow 0$ with keeping $r_{1}>r_{2}$. Then, after eliminating irrelevant factors, we have the equation

$$
c^{3}-5 c^{2}+8 c-5=0,
$$

whose unique real root $\bar{c}$ is approximately 2.75 . Going back to the equations of (B), (C), and (D) and taking $r_{2} \rightarrow 0$ followed by $r_{1} \rightarrow 0$, we derive

$$
\bar{b}_{1}=\frac{\bar{c}^{2}-3 \bar{c}+3}{\bar{c}^{2}-\bar{c}+1} \approx 0.40
$$

and

$$
\bar{b}_{2}=\frac{\bar{c}^{2}-2 \bar{c}+2}{\bar{c}^{2}-\bar{c}+1} \approx 0.70 .
$$


From the equation system of (B), we have the best possible strategy of type (b): $\left(\bar{x}_{0}, \bar{x}_{1}, \bar{x}_{2}, \bar{x}_{3}\right) \rightarrow$ $\left(0, \frac{\bar{b}_{1}}{\bar{c}-1}, \infty, \infty\right) \approx(0,0.23, \infty, \infty)$. From $(\mathrm{C})$, we have the best possible strategy of type $(\mathrm{c})$ : $\left(\bar{x}_{0}, \bar{x}_{1}, \bar{x}_{2}, \bar{x}_{3}\right) \rightarrow\left(0, \frac{\bar{b}_{2}}{\bar{c}-1}, \frac{\bar{b}_{2}}{\bar{c}-1}, \infty\right) \approx(0,0.40,0.40, \infty)$. From (D), we have the best possible strategy of type $(\mathrm{d}):\left(\bar{x}_{0}, \bar{x}_{1}, \bar{x}_{2}, \bar{x}_{3}\right) \rightarrow\left(0, \frac{\bar{b}_{1}}{\bar{c}-1}, \infty, \infty\right) \approx(0,0.23, \infty, \infty)$.

\section{Subclasses of the Multislope Ski-Rental Problem}

In this section we consider the infimum and supremum of the best possible competitive ratio over subclasses of instances, that is, additive instances and investment instances. We will see that the best possible competitive ratio of the hardest additive instance coincides with that of the easiest investment instance, which equals to two.

\subsection{Additive Instance}

Theorem 1 and Corollary 2 provide the infimum of $\tilde{c}(\boldsymbol{r}, \boldsymbol{b})$ over $I_{A}(k)$ as well. Recall that the infimum is achieved by an additive instance.

Corollary 5. It holds $\inf \left\{\tilde{c}(\boldsymbol{r}, \boldsymbol{b}) \mid(\boldsymbol{r}, \boldsymbol{b}) \in I_{A}(k)\right\}=\min \left\{\tilde{c}(\boldsymbol{r}, \boldsymbol{b}) \mid(\boldsymbol{r}, \boldsymbol{b}) \in I_{A}(k)\right\}=\frac{(k+1)^{k}}{(k+1)^{k}-k^{k}}$.

Corollary 6. It holds $\inf \left\{\tilde{c}(\boldsymbol{r}, \boldsymbol{b}) \mid(\boldsymbol{r}, \boldsymbol{b}) \in I_{A}(k), k \geq 2\right\}=\frac{e}{e-1}$.

We establish a supremum of $\tilde{c}(\boldsymbol{r}, \boldsymbol{b})$ for $I_{A}(k)$ with the help of an existing result. In the next theorem we present an instance for which any strategy cannot exceed $(2-\varepsilon)$-competitive. Irani et al. [ISG03] provided the Lower Envelope strategy that is 2-competitive. Note that unlike the case of general instances, the resulting supremum value is constant regardless of $k$.

Theorem 6. Fix $k \geq 2$. For any $\varepsilon \in(0,1)$, there is an additive instance $(\boldsymbol{r}, \boldsymbol{b}) \in I_{A}(k)$ for which no strategy is better than $(2-\varepsilon)$-competitive.

Proof. Given $\varepsilon$, we construct an additive instance $(\boldsymbol{r}, \boldsymbol{b}) \in I_{A}(k)$ by setting $1=r_{0}>r_{1}>\cdots>$ $r_{k-1}=1-\varepsilon, r_{k}=0, b_{0, i}=1-r_{i}$ for $0 \leq i \leq k$, and $b_{i, j}=b_{0, j}-b_{0, i}$ for $0 \leq i<j \leq k$. This instance simulates the classical (i.e., 2-slope) ski-rental problem.

Fix a strategy $\boldsymbol{x} \in S$ arbitrarily. By comparison of the online and offline costs immediately before the transition to state $k$, it follows that

$$
O N\left(\boldsymbol{x}, x_{k}\right)-b_{k-1, k} \geq O P T\left(x_{k}\right) .
$$

Otherwise, the offline optimality fails.

If $x_{k}>1$, we have

$$
O N\left(\boldsymbol{x}, x_{k}\right) \geq O P T\left(x_{k}\right)+b_{k-1, k}=O F F_{k}\left(x_{k}\right)+(1-\varepsilon)=(2-\varepsilon) O F F_{k}\left(x_{k}\right),
$$

since $O P T\left(x_{k}\right)=O F F_{k}\left(x_{k}\right)=1$. On the other hand, if $x_{k} \leq 1$, similarly by $O P T\left(x_{k}\right)=$ $O F F_{0}\left(x_{k}\right)=x_{k}$, we have

$$
O N\left(\boldsymbol{x}, x_{k}\right) \geq O P T\left(x_{k}\right)+b_{k-1, k}=O F F_{0}\left(x_{k}\right)+(1-\varepsilon) \geq(2-\varepsilon) O F F_{0}\left(x_{k}\right) .
$$

Thus, strategy $\boldsymbol{x}$ is shown to be no better than $(2-\varepsilon)$-competitive.

Theorem 7 ([ISG03]). Fix $k \geq 2$. For any additive instance $(\boldsymbol{r}, \boldsymbol{b}) \in I_{A}(k)$, there is a 2competitive strategy.

Corollary 7. For any $k \geq 2, \sup \left\{\tilde{c}(\boldsymbol{r}, \boldsymbol{b}) \mid(\boldsymbol{r}, \boldsymbol{b}) \in I_{A}(k)\right\}=2$. 


\subsection{Investment Instance}

The next theorem states that the value of the infimum of $\tilde{c}(\boldsymbol{r}, \boldsymbol{b})$ over $I_{I}(k)$ is independent of $k$.

Theorem 8. For any $k \geq 2, \inf \left\{\tilde{c}(\boldsymbol{r}, \boldsymbol{b}) \mid(\boldsymbol{r}, \boldsymbol{b}) \in I_{I}(k)\right\}=\min \left\{\tilde{c}(\boldsymbol{r}, \boldsymbol{b}) \mid(\boldsymbol{r}, \boldsymbol{b}) \in I_{I}(k)\right\}=2$. The strategy and the instance that achieve the minimum are: $\bar{x}_{0}=0$ and $\bar{x}_{1}=\bar{x}_{2}=\cdots=\bar{x}_{k}=$ $1 ; \overline{\boldsymbol{r}}$ is such that $1=\bar{r}_{0}>\bar{r}_{1}>\cdots>\bar{r}_{k}=0 ; \bar{b}_{i, j}=1-\bar{r}_{j}$ for $0 \leq i<j \leq k$.

Proof. With a simple calculation we confirm that $\overline{\boldsymbol{x}} \in S$ and $(\overline{\boldsymbol{r}}, \overline{\boldsymbol{b}}) \in I_{I}(k)$, and that $\overline{\boldsymbol{x}}$ is 2-competitive for $(\overline{\boldsymbol{r}}, \overline{\boldsymbol{b}})$.

The remainder is to prove that for any instance, the competitive ratio of any strategy is no smaller than 2. Fix a strategy $\boldsymbol{x} \in S$ and an instance $(\boldsymbol{r}, \boldsymbol{b}) \in I_{I}(k)$ arbitrarily. Observe that even immediately before the transition time $x_{k}$, the optimal offline cost does not exceed the cost of $\boldsymbol{x}$. That is to say, $O N\left(\boldsymbol{x}, x_{k}\right)-1 \geq O P T\left(x_{k}\right)$ holds. Note here that the transition cost to the last state $k$ is one, regardless of the previous state. In addition, the optimal offline player never incurs more than a cost of unity. Therefore,

$$
O N\left(\boldsymbol{x}, x_{k}\right) \geq O P T\left(x_{k}\right)+1 \geq 2 O P T\left(x_{k}\right),
$$

which implies that strategy $\boldsymbol{x}$ cannot be better than 2-competitive.

We remark that all of the suprema given by Theorems $2,3,4$, and 5 are originally proved for investment instances.

Corollary 8. It holds that $\sup \left\{\tilde{c}(\boldsymbol{r}, \boldsymbol{b}) \mid(\boldsymbol{r}, \boldsymbol{b}) \in I_{I}(2)\right\}=\bar{c}$, where $\overline{\boldsymbol{c}}$ is the unique real root of $c^{3}-4 c^{2}+5 c-3=0$, approximately 2.47 .

Corollary 9. It holds that $\sup \left\{\tilde{c}(\boldsymbol{r}, \boldsymbol{b}) \mid(\boldsymbol{r}, \boldsymbol{b}) \in I_{I}(3)\right\}=\bar{c}$, where $\bar{c}$ is the unique real root of $c^{3}-5 c^{2}+8 c-5=0$, approximately 2.75 .

Corollary 10 ([Dam03]). It holds $\sup \left\{\tilde{c}(\boldsymbol{r}, \boldsymbol{b}) \mid(\boldsymbol{r}, \boldsymbol{b}) \in I_{I}(k), k \geq 2\right\} \geq(5+\sqrt{5}) / 2 \approx 3.62$.

Corollary 11 ([BCN00]). It holds $\sup \left\{\tilde{c}(\boldsymbol{r}, \boldsymbol{b}) \mid(\boldsymbol{r}, \boldsymbol{b}) \in I_{I}(k), k \geq 2\right\} \leq 4$.

\section{Concluding Remarks}

In this paper we have settled the problem of finding the infimum of the best possible competitive ratio. As in Corollary 1, the infimum value is explicitly presented in terms of the number of states. If it is allowed to freely design both a strategy and an instance, one can enjoy a competitive ratio of that value.

In contrast, many problems concerning the supremum of the best possible competitive ratio are left open. Although we have presented suprema for the two problems where there are three or four states, our current technique does not seem powerful enough for finding a supremum for each of the 5-or-more-state problems in general. It is also interesting to bound or obtain the supremum for the problem where arbitrary number of states are available, which is so far known to lie between $(5+\sqrt{5}) / 2 \approx 3.62$ and 4 .

\section{References}

[AIS08] J. Augustine, S. Irani, and C. Swamy. Optimal power-down strategies. SIAM J. Comput., 37(5):1499-1516, 2008. 
[BCN00] Y. Bejerano, I. Cidon, and J. Naor. Dynamic session management for static and mobile users: a competitive on-line algorithmic approach. In Proc. DIAL-M '00, pages $65-74,2000$.

[Dam03] P. Damaschke. Nearly optimal strategies for special cases of on-line capital investment. Theor. Comput. Sci., 302(1-3):35-44, 2003.

[EYKL99] R. El-Yaniv, R. Kaniel, and N. Linial. Competitive optimal on-line leasing. Algorithmica, 25(1):116-140, 1999.

[Fle01] R. Fleischer. On the Bahncard problem. Theoret. Comput. Sci., 268(1):161-174, 2001.

[ISG03] S. Irani, S. Shukla, and R. Gupta. Online strategies for dynamic power management in systems with multiple power-saving states. ACM Trans. Embed. Comput. Syst., $2(3): 325-346,2003$.

[Kar96] A. R. Karlin. On the performance of competitive algorithms in practice. In A. Fiat and G. J. Woeginger, editors, Online Algorithms, volume 1442 of LNCS, pages 373-384. Springer, 1996.

[KKR01] A. R. Karlin, C. Kenyon, and D. Randall. Dynamic TCP acknowledgement and other stories about $e /(e-1)$. In Proc. STOC '01, pages 502-509, 2001.

[KMMO94] A. R. Karlin, M. S. Manasse, L. McGeogh, and S. Owicki. Competitive randomized algorithms for nonuniform problems. Algorithmica, 11(6):542-571, 1994.

[KMRS88] A. R. Karlin, M. S. Manasse, L. Rudolph, and D. D. Sleator. Competitive snoopy caching. Algorithmica, 3:77-119, 1988.

[LPSR08] Z. Lotker, B. Patt-Shamir, and D. Rawitz. Rent, lease or buy: Randomized algorithms for multislope ski rental. In Proc. STACS '08, pages 503-514, 2008. 